\title{
Tables of Genera of Groups of Linear Fractional Transformations*
}

\author{
Harriet Fell, Morris Newman, and Edward Ordman
}

(October 29, 1962)

\begin{abstract}
The genera of the groups $\Gamma_{0}(n), \Gamma^{*}(n)$ together with certain associated number-theoretic functions are given for $1 \leq n \leq 1000$.
\end{abstract}

The $2 \times 2$ modular group $\Gamma$ and its subgroups are of fundamental importance in the theory of automorphic functions (in particular the elliptic modular functions) and in the theory of Riemann surfaces. Let $G$ be such a subgroup. Then the substitutions

$$
\tau^{\prime}=\frac{a \tau+b}{c \tau+d}
$$

of $G$ map the upper $\tau$ half-plane onto itself, and the points of this half-plane are partitioned into equivalence classes modulo $G$. A set of points consisting of one point from each equivalence class is termed a fundamental set, and if $G$ is of finite index in $\Gamma$ there is a simple way of selecting a standard fundamental set $R$, which is fully described in Ford's book [1] ${ }^{1}$ or in Gunning's book [3]. The set $R$ is commonly called a fundamental region. After appropriate identifications of sides and vertices are made $R$ becomes a surface whose genus $g$ plays a central role in the study of $G$.

Among the subgroups of finite index in $\Gamma$ the congruence subgroups have been studied most extensively and among the congruence subgroups the (nonnormal) subgroups $\Gamma_{0}(n)$ are of primary importance. These are defined for every natural number $n$ as the totality of substitutions (1) where $a$, $b, c, d$ are rational integers, $a d-b c=1$, and $c \equiv 0$ $(\bmod n)$.

If the substitution

$$
\tau^{\prime}=-\frac{1}{n \tau}
$$

is adjoined to $\Gamma_{0}(n)$ the larger group so obtained (in general not a subgroup of $\Gamma$ ) is donoted by $\Gamma^{*}(n)$. Formulas for the genera $g_{0}(n), g^{*}(n)$ of $\Gamma_{0}(n), \Gamma^{*}(n)$, respectively, have been given by $\mathrm{F}$. Klein [6], E. Hecke [4,5], and R. Fricke [2]. Denote the number of solutions of the congruence

$$
x^{2}-x+1 \equiv 0(\bmod n), 0 \leq x \leq n-1
$$

by $\nu_{1}(n)$, and the number of solutions of the congruence

*The computation of the tables was carried out by the first and third authors at the suggestion of the second author, as a summer project at NBS. The work was supported by the Office of Naval Research.

1 Figures in brackets indicate the literature references at the end of this paper.

$$
x^{2}+1 \equiv 0(\bmod n), 0 \leq x \leq n-1
$$

by $\nu_{2}(n)$. Set

$$
\begin{gathered}
\mu(n)=n \prod_{q \mid n}\left(1+\frac{1}{q}\right), \\
\sigma_{0}(n)=\sum_{d \mid n} \varphi\left(d, \frac{n}{d}\right)=\prod_{q^{e} \mid n}\left(g^{\left[\frac{e}{2}\right]}+q^{\left[\frac{e-1}{2}\right]}\right),
\end{gathered}
$$

where $\left(d, \frac{n}{d}\right)$ denotes the greatest common divisor of $d$ and $\frac{n}{d}$, and $\varphi$ is the Euler function. Then $\nu_{1}(n)$ is the number of inequivalent elliptic vertices of period 3 of the fundamental region $R_{n}$ of $\Gamma_{0}(n), \nu_{2}(n)$ the number of inequivalent elliptic vertices of period 2 of $R_{n}, \mu(n)$ the index of $\Gamma_{0}(n)$ in $\Gamma$, and $\sigma_{0}(n)$ the number of inequivalent parabolic vertices of $R_{n}$. The genus $g_{0}(n)$ of $\Gamma_{0}(n)$ is then

$$
g_{0}(n)=1+\frac{\mu(n)}{12}-\frac{\nu_{1}(n)}{3}-\frac{\nu_{2}(n)}{4}-\frac{\sigma_{0}(n)}{2} .
$$

Furthermore let $h(-4 n)$ denote the number of classes of primitive positive binary quadratic forms of discriminant $-4 n$, and set

$$
\delta_{n}=\left\{\begin{array}{l}
2 n \equiv 7(\bmod 8) \\
\frac{4}{3} n \equiv 3(\bmod 8), n>3 \\
1 \text { otherwise. }
\end{array}\right.
$$

Then the genus $g^{*}(n)$ of $\Gamma^{*}(n)$ is given by

$$
g^{*}(n)=\frac{1}{2} g_{0}(n)+\frac{1}{2}-\frac{1}{4} \delta_{n} h(-4 n) .
$$

In this article we give the actual numerical values of $g_{0}(n), g^{*}(n)$ for $1 \leq n \leq 1000$ together with the values of the associated functions $\nu_{1}(n), \nu_{2}(n), \mu(n)$, $\sigma_{0}(n), \mathrm{h}(-4 n)$. These were computed on the IBM 7090 of the NBS in a negligible amount of time. The resulting tables are of considerable interest however and should prove useful in many number- 
theoretic investigations, as well as in the study of the groups $\Gamma_{0}(n), \Gamma^{*}(n)$.

We say some words about the computation of the tables. The functions $\nu_{1}(n), \nu_{2}(n)$ were computed directly as the number of solutions of the congruences (2), (3) respectively. It is easy to give closed expressions for $\nu_{1}(n), \nu_{2}(n)$ however. These are

$$
\begin{aligned}
& \nu_{1}(n)=\left\{\begin{array}{l}
02 \mid n \text { or } 9 \mid n \\
\prod_{q \mid n}\left(1+\left(-\frac{3}{q}\right)\right) \text { otherwise, }
\end{array}\right. \\
& \nu_{2}(n)=\left\{\begin{array}{l}
04 \mid n \\
\prod_{q \mid n}\left(1+\left(-\frac{4}{q}\right)\right) \text { otherwise. }
\end{array}\right.
\end{aligned}
$$

We see that (4) and (5) imply that for $n>3, \nu_{1}(n)$ and $\nu_{2}(n)$ can take only 0 or powers of 2 as values. This provides an excellent check on the computation of $\nu_{1}(n), \nu_{2}(n)$.

The function $\mu(n)$ is a multiplicative arithmetic function of $n$ with easily computed values at the prime powers. In addition, if $q$ is a prime $\mu(n)$ satisfies

$$
\mu(n q)=\left\{\begin{array}{l}
q \mu(n) q \mid n \\
(q+1) \mu(n) \text { otherwise. }
\end{array}\right.
$$

Relationship (6) provides an efficient and easily applied check on the computation of $\mu(n)$. Similar remarks apply to the function $\sigma_{0}(n)$.

The class number $h(-4 n)$ was computed as the number of solutions of the system

$$
\left\{\begin{array}{l}
b^{2}-4 a c=-4 n \\
(a, b, c)=1 \\
-a<b \leq a<c \text { or } 0 \leq b \leq a=c .
\end{array}\right.
$$

A check on the computation was provided by determining the parity of $h(-4 n)$. It is not difficult to show from (7) that

where

$$
h(-4 n) \equiv U(n)+V(n) \quad(\bmod 2),
$$

$$
U(n)=\sum_{d \mid n} 1
$$$$
\left(d, \frac{n}{d}\right)=1
$$$$
d^{2}<n
$$

$$
V(n)=\sum_{d \mid n} 1
$$

$$
\begin{aligned}
& \left(d, \frac{d+\frac{n}{d}}{2}\right)=1 \\
& d^{2} \leq n \\
& d+\frac{n}{d} \equiv 2(\bmod 4) .
\end{aligned}
$$

From (9) and (10) it follows for example that for $n>1, n \equiv 1(\bmod 4) h(-4 n)$ is even. For $n \equiv 3(\bmod$ 4) $h(-4 n)$ is even if $n$ is not a prime power and odd if $n$ is a prime power. Another excellent check arises from the fact that for $n \equiv 3(\bmod 8), n>3$, $h(-4 n)$ is divisible by 3 .

A different type of check is available from the relationship

\begin{tabular}{|c|c|c|c|c|c|c|c|}
\hline$n$ & $\nu_{1}(n)$ & $\nu_{2}(n)$ & $\mu(n)$ & $\boldsymbol{\sigma}_{0}(n)$ & $h(-4 n)$ & $g_{0}(n)$ & $g^{*}(n)$ \\
\hline $\begin{array}{l}1 \\
2 \\
3 \\
4 \\
5\end{array}$ & $\begin{array}{l}1 \\
0 \\
1 \\
0 \\
0\end{array}$ & $\begin{array}{l}1 \\
1 \\
0 \\
0 \\
2\end{array}$ & $\begin{array}{l}1 \\
3 \\
4 \\
6 \\
6\end{array}$ & $\begin{array}{l}1 \\
2 \\
2 \\
3 \\
2\end{array}$ & $\begin{array}{l}1 \\
1 \\
1 \\
1 \\
2\end{array}$ & $\begin{array}{l}0 \\
0 \\
0 \\
0 \\
0\end{array}$ & $\begin{array}{l}0 \\
0 \\
0 \\
0 \\
0\end{array}$ \\
\hline $\begin{array}{r}6 \\
7 \\
8 \\
9 \\
10\end{array}$ & $\begin{array}{l}0 \\
2 \\
0 \\
0 \\
0\end{array}$ & $\begin{array}{l}0 \\
0 \\
0 \\
0 \\
2\end{array}$ & $\begin{array}{r}12 \\
8 \\
12 \\
12 \\
18\end{array}$ & $\begin{array}{l}4 \\
2 \\
4 \\
4 \\
4\end{array}$ & $\begin{array}{l}2 \\
1 \\
2 \\
2 \\
2\end{array}$ & $\begin{array}{l}0 \\
0 \\
0 \\
0 \\
0\end{array}$ & $\begin{array}{l}0 \\
0 \\
0 \\
0 \\
0\end{array}$ \\
\hline $\begin{array}{l}11 \\
12 \\
13 \\
14 \\
15\end{array}$ & $\begin{array}{l}0 \\
0 \\
2 \\
0 \\
0\end{array}$ & $\begin{array}{l}0 \\
0 \\
2 \\
0 \\
0\end{array}$ & $\begin{array}{l}12 \\
24 \\
14 \\
24 \\
24\end{array}$ & $\begin{array}{l}2 \\
6 \\
2 \\
4 \\
4\end{array}$ & $\begin{array}{l}3 \\
2 \\
2 \\
4 \\
2\end{array}$ & $\begin{array}{l}1 \\
0 \\
0 \\
1 \\
1\end{array}$ & $\begin{array}{l}0 \\
0 \\
0 \\
0 \\
0\end{array}$ \\
\hline $\begin{array}{l}16 \\
17 \\
18 \\
19 \\
20\end{array}$ & $\begin{array}{l}0 \\
0 \\
0 \\
2 \\
0\end{array}$ & $\begin{array}{l}0 \\
2 \\
0 \\
0 \\
0\end{array}$ & $\begin{array}{l}24 \\
18 \\
36 \\
20 \\
36\end{array}$ & $\begin{array}{l}6 \\
2 \\
8 \\
2 \\
6\end{array}$ & $\begin{array}{l}2 \\
4 \\
2 \\
3 \\
4\end{array}$ & $\begin{array}{l}0 \\
1 \\
0 \\
1 \\
1\end{array}$ & $\begin{array}{l}0 \\
0 \\
0 \\
0 \\
0\end{array}$ \\
\hline $\begin{array}{l}21 \\
22 \\
23 \\
24 \\
25\end{array}$ & $\begin{array}{l}2 \\
0 \\
0 \\
0 \\
0\end{array}$ & $\begin{array}{l}0 \\
0 \\
0 \\
0 \\
2\end{array}$ & $\begin{array}{l}32 \\
36 \\
24 \\
48 \\
30\end{array}$ & $\begin{array}{l}4 \\
4 \\
2 \\
8 \\
6\end{array}$ & $\begin{array}{l}4 \\
2 \\
3 \\
4 \\
2\end{array}$ & $\begin{array}{l}1 \\
2 \\
2 \\
1 \\
0\end{array}$ & $\begin{array}{l}0 \\
1 \\
0 \\
0 \\
0\end{array}$ \\
\hline $\begin{array}{l}26 \\
27 \\
28 \\
29 \\
30\end{array}$ & $\begin{array}{l}0 \\
0 \\
0 \\
0 \\
0\end{array}$ & $\begin{array}{l}2 \\
0 \\
0 \\
2 \\
0\end{array}$ & $\begin{array}{l}42 \\
36 \\
48 \\
30 \\
72\end{array}$ & $\begin{array}{l}4 \\
6 \\
6 \\
2 \\
8\end{array}$ & $\begin{array}{l}6 \\
3 \\
2 \\
6 \\
4\end{array}$ & $\begin{array}{l}2 \\
1 \\
2 \\
2 \\
3\end{array}$ & $\begin{array}{l}0 \\
0 \\
1 \\
0 \\
1\end{array}$ \\
\hline $\begin{array}{l}31 \\
32 \\
33 \\
34 \\
35\end{array}$ & $\begin{array}{l}2 \\
0 \\
0 \\
0 \\
0\end{array}$ & $\begin{array}{l}0 \\
0 \\
0 \\
2 \\
0\end{array}$ & $\begin{array}{l}32 \\
48 \\
48 \\
54 \\
48\end{array}$ & $\begin{array}{l}2 \\
8 \\
4 \\
4 \\
4\end{array}$ & $\begin{array}{l}3 \\
4 \\
4 \\
4 \\
6\end{array}$ & $\begin{array}{l}2 \\
1 \\
3 \\
3 \\
3\end{array}$ & $\begin{array}{l}0 \\
0 \\
1 \\
1 \\
0\end{array}$ \\
\hline $\begin{array}{l}36 \\
37 \\
38 \\
39 \\
40\end{array}$ & $\begin{array}{l}0 \\
2 \\
0 \\
2 \\
0\end{array}$ & $\begin{array}{l}0 \\
2 \\
0 \\
0 \\
0\end{array}$ & $\begin{array}{l}72 \\
38 \\
60 \\
56 \\
72\end{array}$ & $\begin{array}{r}12 \\
2 \\
4 \\
4 \\
8\end{array}$ & $\begin{array}{l}4 \\
2 \\
6 \\
4 \\
4\end{array}$ & $\begin{array}{l}1 \\
2 \\
4 \\
3 \\
3\end{array}$ & $\begin{array}{l}0 \\
1 \\
1 \\
0 \\
1\end{array}$ \\
\hline $\begin{array}{l}41 \\
42 \\
43 \\
44 \\
45\end{array}$ & $\begin{array}{l}0 \\
0 \\
2 \\
0 \\
0\end{array}$ & $\begin{array}{l}2 \\
0 \\
0 \\
0 \\
0\end{array}$ & $\begin{array}{l}42 \\
96 \\
44 \\
72 \\
72\end{array}$ & $\begin{array}{l}2 \\
8 \\
2 \\
6 \\
8\end{array}$ & $\begin{array}{l}8 \\
4 \\
3 \\
6 \\
4\end{array}$ & $\begin{array}{l}3 \\
5 \\
3 \\
4 \\
3\end{array}$ & $\begin{array}{l}0 \\
2 \\
1 \\
1 \\
1\end{array}$ \\
\hline $\begin{array}{l}46 \\
47 \\
48 \\
49 \\
50\end{array}$ & $\begin{array}{l}0 \\
0 \\
0 \\
2 \\
0\end{array}$ & $\begin{array}{l}0 \\
0 \\
0 \\
0 \\
2\end{array}$ & $\begin{array}{l}72 \\
48 \\
96 \\
56 \\
90\end{array}$ & $\begin{array}{r}4 \\
2 \\
12 \\
8 \\
12\end{array}$ & $\begin{array}{l}4 \\
5 \\
4 \\
4 \\
6\end{array}$ & $\begin{array}{l}5 \\
4 \\
3 \\
1 \\
2\end{array}$ & $\begin{array}{l}2 \\
0 \\
1 \\
0 \\
0\end{array}$ \\
\hline $\begin{array}{l}51 \\
52 \\
53 \\
54 \\
55\end{array}$ & $\begin{array}{l}0 \\
0 \\
0 \\
0 \\
0\end{array}$ & $\begin{array}{l}0 \\
0 \\
2 \\
0 \\
0\end{array}$ & $\begin{array}{r}72 \\
84 \\
54 \\
108 \\
72\end{array}$ & $\begin{array}{r}4 \\
6 \\
2 \\
12 \\
4\end{array}$ & $\begin{array}{l}6 \\
4 \\
6 \\
6 \\
4\end{array}$ & $\begin{array}{l}5 \\
5 \\
4 \\
4 \\
5\end{array}$ & $\begin{array}{l}1 \\
2 \\
1 \\
1 \\
1\end{array}$ \\
\hline
\end{tabular}

$$
h\left(-4 f m^{2}\right)=h(-4 f) m \prod_{p \mid m}\left\{1-\frac{1}{p}\left(\frac{-4 f}{p}\right)\right\},
$$

where $f>1, f \equiv 1,2(\bmod 4)$ and square-free.

The computation of $g_{\mathrm{o}}(n)$ was checked by computing $12 g_{0}(n)$ and verifying the divisibility of 12 $g_{0}(n)$ by 12 . The computation of $g^{*}(n)$ was checked by the fact that always, $g^{*}(n) \leq 1 / 2 g(n)$. In addition, R. Fricke gives in [2] the first 50 or so values of $g_{0}(n), g^{*}(n)$ and these agree with the values given here. 


\begin{tabular}{|c|c|c|c|c|c|c|c|c|c|c|c|c|c|c|c|}
\hline$n$ & $\nu_{1}(n)$ & $\nu_{2}(n)$ & $\mu(n)$ & $\sigma_{0}(n)$ & $h(-4 n)$ & $g_{0}(n)$ & $g^{*}(n)$ & $n$ & $\nu_{1}(n)$ & $\nu_{2}(n)$ & $\mu(n)$ & $\sigma_{0}(n)$ & $h(-4 n)$ & $g_{0}(n)$ & $g^{*}(n)$ \\
\hline $\begin{array}{l}56 \\
57 \\
58 \\
59 \\
60\end{array}$ & $\begin{array}{l}0 \\
2 \\
0 \\
0 \\
0\end{array}$ & $\begin{array}{l}0 \\
0 \\
2 \\
0 \\
0\end{array}$ & $\begin{array}{r}96 \\
80 \\
90 \\
60 \\
144\end{array}$ & $\begin{array}{r}8 \\
4 \\
4 \\
2 \\
12\end{array}$ & $\begin{array}{l}8 \\
4 \\
2 \\
9 \\
4\end{array}$ & $\begin{array}{l}5 \\
5 \\
6 \\
5 \\
7\end{array}$ & $\begin{array}{l}1 \\
2 \\
3 \\
0 \\
3\end{array}$ & $\begin{array}{l}136 \\
137 \\
138 \\
139 \\
140\end{array}$ & $\begin{array}{l}0 \\
0 \\
0 \\
2 \\
0\end{array}$ & $\begin{array}{l}0 \\
2 \\
0 \\
0 \\
0\end{array}$ & $\begin{array}{l}216 \\
138 \\
288 \\
140 \\
288\end{array}$ & $\begin{array}{r}8 \\
2 \\
8 \\
2 \\
12\end{array}$ & $\begin{array}{r}8 \\
8 \\
8 \\
9 \\
12\end{array}$ & $\begin{array}{l}15 \\
11 \\
21 \\
11 \\
19\end{array}$ & $\begin{array}{l}6 \\
4 \\
9 \\
3 \\
7\end{array}$ \\
\hline $\begin{array}{l}61 \\
62 \\
63 \\
64 \\
65\end{array}$ & $\begin{array}{l}2 \\
0 \\
0 \\
0 \\
0\end{array}$ & $\begin{array}{l}2 \\
0 \\
0 \\
0 \\
4\end{array}$ & $\begin{array}{l}62 \\
96 \\
96 \\
96 \\
84\end{array}$ & $\begin{array}{r}2 \\
4 \\
8 \\
12 \\
4\end{array}$ & $\begin{array}{l}6 \\
8 \\
4 \\
4 \\
8\end{array}$ & $\begin{array}{l}4 \\
7 \\
5 \\
3 \\
5\end{array}$ & $\begin{array}{l}1 \\
2 \\
1 \\
1 \\
1\end{array}$ & $\begin{array}{l}141 \\
142 \\
143 \\
144 \\
145\end{array}$ & $\begin{array}{l}0 \\
0 \\
0 \\
0 \\
0\end{array}$ & $\begin{array}{l}0 \\
0 \\
0 \\
0 \\
4\end{array}$ & $\begin{array}{l}192 \\
216 \\
168 \\
288 \\
180\end{array}$ & $\begin{array}{r}4 \\
4 \\
4 \\
24 \\
4\end{array}$ & $\begin{array}{r}8 \\
4 \\
10 \\
8 \\
8\end{array}$ & $\begin{array}{l}15 \\
17 \\
13 \\
13 \\
13\end{array}$ & $\begin{array}{l}6 \\
8 \\
2 \\
5 \\
5\end{array}$ \\
\hline $\begin{array}{l}66 \\
67 \\
68 \\
69 \\
70\end{array}$ & $\begin{array}{l}0 \\
2 \\
0 \\
0 \\
0\end{array}$ & $\begin{array}{l}0 \\
0 \\
0 \\
0 \\
0\end{array}$ & $\begin{array}{r}144 \\
68 \\
108 \\
96 \\
144\end{array}$ & $\begin{array}{l}8 \\
2 \\
6 \\
4 \\
8\end{array}$ & $\begin{array}{l}8 \\
3 \\
8 \\
8 \\
4\end{array}$ & $\begin{array}{l}9 \\
5 \\
7 \\
7 \\
9\end{array}$ & $\begin{array}{l}3 \\
2 \\
2 \\
2 \\
4\end{array}$ & $\begin{array}{l}146 \\
147 \\
148 \\
149 \\
150\end{array}$ & $\begin{array}{l}0 \\
2 \\
0 \\
0 \\
0\end{array}$ & $\begin{array}{l}2 \\
0 \\
0 \\
2 \\
0\end{array}$ & $\begin{array}{l}222 \\
224 \\
228 \\
150 \\
360\end{array}$ & $\begin{array}{r}4 \\
16 \\
6 \\
2 \\
24\end{array}$ & $\begin{array}{r}16 \\
6 \\
4 \\
14 \\
8\end{array}$ & $\begin{array}{l}17 \\
11 \\
17 \\
12 \\
19\end{array}$ & $\begin{array}{l}5 \\
4 \\
8 \\
3 \\
8\end{array}$ \\
\hline $\begin{array}{l}71 \\
72 \\
73 \\
74 \\
75\end{array}$ & $\begin{array}{l}0 \\
0 \\
2 \\
0 \\
0\end{array}$ & $\begin{array}{l}0 \\
0 \\
2 \\
2 \\
0\end{array}$ & $\begin{array}{r}72 \\
144 \\
74 \\
114 \\
120\end{array}$ & $\begin{array}{r}2 \\
16 \\
2 \\
4 \\
12\end{array}$ & $\begin{array}{r}7 \\
4 \\
4 \\
10 \\
6\end{array}$ & $\begin{array}{l}6 \\
5 \\
5 \\
8 \\
5\end{array}$ & $\begin{array}{l}0 \\
2 \\
2 \\
2 \\
1\end{array}$ & $\begin{array}{l}151 \\
152 \\
153 \\
154 \\
155\end{array}$ & $\begin{array}{l}2 \\
0 \\
0 \\
0 \\
0\end{array}$ & $\begin{array}{l}0 \\
0 \\
0 \\
0 \\
0\end{array}$ & $\begin{array}{l}152 \\
240 \\
216 \\
288 \\
192\end{array}$ & $\begin{array}{l}2 \\
8 \\
8 \\
8 \\
4\end{array}$ & $\begin{array}{r}7 \\
12 \\
8 \\
8 \\
12\end{array}$ & $\begin{array}{l}12 \\
17 \\
15 \\
21 \\
15\end{array}$ & $\begin{array}{l}3 \\
6 \\
6 \\
9 \\
4\end{array}$ \\
\hline $\begin{array}{l}76 \\
77 \\
78 \\
79 \\
80\end{array}$ & $\begin{array}{l}0 \\
0 \\
0 \\
2 \\
0\end{array}$ & $\begin{array}{l}0 \\
0 \\
0 \\
0 \\
0\end{array}$ & $\begin{array}{r}120 \\
96 \\
168 \\
80 \\
144\end{array}$ & $\begin{array}{r}6 \\
4 \\
8 \\
2 \\
12\end{array}$ & $\begin{array}{l}6 \\
8 \\
4 \\
5 \\
8\end{array}$ & $\begin{array}{r}8 \\
7 \\
11 \\
6 \\
7\end{array}$ & $\begin{array}{l}3 \\
2 \\
5 \\
1 \\
2\end{array}$ & $\begin{array}{l}156 \\
157 \\
158 \\
159 \\
160\end{array}$ & $\begin{array}{l}0 \\
2 \\
0 \\
0 \\
0\end{array}$ & $\begin{array}{l}0 \\
2 \\
0 \\
0 \\
0\end{array}$ & $\begin{array}{l}336 \\
158 \\
240 \\
216 \\
288\end{array}$ & $\begin{array}{r}12 \\
2 \\
4 \\
4 \\
16\end{array}$ & $\begin{array}{r}8 \\
6 \\
8 \\
10 \\
8\end{array}$ & $\begin{array}{l}23 \\
12 \\
19 \\
17 \\
17\end{array}$ & $\begin{array}{r}10 \\
5 \\
8 \\
4 \\
7\end{array}$ \\
\hline $\begin{array}{l}81 \\
82 \\
83 \\
84 \\
85\end{array}$ & $\begin{array}{l}0 \\
0 \\
0 \\
0 \\
0\end{array}$ & $\begin{array}{l}0 \\
2 \\
0 \\
0 \\
4\end{array}$ & $\begin{array}{r}108 \\
126 \\
84 \\
192 \\
108\end{array}$ & $\begin{array}{r}12 \\
4 \\
2 \\
12 \\
4\end{array}$ & $\begin{array}{l}6 \\
4 \\
9 \\
8 \\
4\end{array}$ & $\begin{array}{r}4 \\
9 \\
7 \\
11 \\
7\end{array}$ & $\begin{array}{l}1 \\
4 \\
1 \\
4 \\
3\end{array}$ & $\begin{array}{l}161 \\
162 \\
163 \\
164 \\
165\end{array}$ & $\begin{array}{l}0 \\
0 \\
2 \\
0 \\
0\end{array}$ & $\begin{array}{l}0 \\
0 \\
0 \\
0 \\
0\end{array}$ & $\begin{array}{l}192 \\
324 \\
164 \\
252 \\
288\end{array}$ & $\begin{array}{r}4 \\
24 \\
2 \\
6 \\
8\end{array}$ & $\begin{array}{r}16 \\
6 \\
3 \\
16 \\
8\end{array}$ & $\begin{array}{l}15 \\
16 \\
13 \\
19 \\
21\end{array}$ & $\begin{array}{l}4 \\
7 \\
6 \\
6 \\
9\end{array}$ \\
\hline $\begin{array}{l}86 \\
87 \\
88 \\
89 \\
90\end{array}$ & $\begin{array}{l}0 \\
0 \\
0 \\
0 \\
0\end{array}$ & $\begin{array}{l}0 \\
0 \\
0 \\
2 \\
0\end{array}$ & $\begin{array}{r}132 \\
120 \\
144 \\
90 \\
216\end{array}$ & $\begin{array}{r}4 \\
4 \\
8 \\
2 \\
16\end{array}$ & $\begin{array}{r}10 \\
6 \\
4 \\
12 \\
8\end{array}$ & $\begin{array}{r}10 \\
9 \\
9 \\
7 \\
11\end{array}$ & $\begin{array}{l}3 \\
2 \\
4 \\
1 \\
4\end{array}$ & $\begin{array}{l}166 \\
167 \\
168 \\
169 \\
170\end{array}$ & $\begin{array}{l}0 \\
0 \\
0 \\
2 \\
0\end{array}$ & $\begin{array}{l}0 \\
0 \\
0 \\
2 \\
4\end{array}$ & $\begin{array}{l}252 \\
168 \\
384 \\
182 \\
324\end{array}$ & $\begin{array}{r}4 \\
2 \\
16 \\
14 \\
8\end{array}$ & $\begin{array}{r}10 \\
11 \\
8 \\
6 \\
12\end{array}$ & $\begin{array}{r}20 \\
14 \\
25 \\
8 \\
23\end{array}$ & $\begin{array}{r}8 \\
2 \\
11 \\
3 \\
9\end{array}$ \\
\hline $\begin{array}{l}91 \\
92 \\
93 \\
94 \\
95\end{array}$ & $\begin{array}{l}4 \\
0 \\
2 \\
0 \\
0\end{array}$ & $\begin{array}{l}0 \\
0 \\
0 \\
0 \\
0\end{array}$ & $\begin{array}{l}112 \\
144 \\
128 \\
144 \\
120\end{array}$ & $\begin{array}{l}4 \\
6 \\
4 \\
4 \\
4\end{array}$ & $\begin{array}{l}6 \\
6 \\
4 \\
8 \\
8\end{array}$ & $\begin{array}{r}7 \\
10 \\
9 \\
11 \\
9\end{array}$ & $\begin{array}{l}2 \\
4 \\
4 \\
4 \\
1\end{array}$ & $\begin{array}{l}171 \\
172 \\
173 \\
174 \\
175\end{array}$ & $\begin{array}{l}0 \\
0 \\
0 \\
0 \\
0\end{array}$ & $\begin{array}{l}0 \\
0 \\
2 \\
0 \\
0\end{array}$ & $\begin{array}{l}240 \\
264 \\
174 \\
360 \\
240\end{array}$ & $\begin{array}{r}8 \\
6 \\
2 \\
8 \\
12\end{array}$ & $\begin{array}{r}12 \\
6 \\
14 \\
12 \\
6\end{array}$ & $\begin{array}{l}17 \\
20 \\
14 \\
27 \\
15\end{array}$ & $\begin{array}{r}5 \\
9 \\
4 \\
11 \\
5\end{array}$ \\
\hline $\begin{array}{r}96 \\
97 \\
98 \\
99 \\
100\end{array}$ & $\begin{array}{l}0 \\
2 \\
0 \\
0 \\
0\end{array}$ & $\begin{array}{l}0 \\
2 \\
0 \\
0 \\
0\end{array}$ & $\begin{array}{r}192 \\
98 \\
168 \\
144 \\
180\end{array}$ & $\begin{array}{r}16 \\
2 \\
16 \\
8 \\
18\end{array}$ & $\begin{array}{l}8 \\
4 \\
8 \\
6 \\
4\end{array}$ & $\begin{array}{l}9 \\
7 \\
7 \\
9 \\
7\end{array}$ & $\begin{array}{l}3 \\
3 \\
2 \\
3 \\
3\end{array}$ & $\begin{array}{l}176 \\
177 \\
178 \\
179 \\
180\end{array}$ & $\begin{array}{l}0 \\
0 \\
0 \\
0 \\
0\end{array}$ & $\begin{array}{l}0 \\
0 \\
2 \\
0 \\
0\end{array}$ & $\begin{array}{l}288 \\
240 \\
270 \\
180 \\
432\end{array}$ & $\begin{array}{r}12 \\
4 \\
4 \\
2 \\
24\end{array}$ & $\begin{array}{r}12 \\
4 \\
8 \\
15 \\
8\end{array}$ & $\begin{array}{l}19 \\
19 \\
21 \\
15 \\
25\end{array}$ & $\begin{array}{r}7 \\
9 \\
9 \\
3 \\
11\end{array}$ \\
\hline $\begin{array}{l}101 \\
102 \\
103 \\
104 \\
105\end{array}$ & $\begin{array}{l}0 \\
0 \\
2 \\
0 \\
0\end{array}$ & $\begin{array}{l}2 \\
0 \\
0 \\
0 \\
0\end{array}$ & $\begin{array}{l}102 \\
216 \\
104 \\
168 \\
192\end{array}$ & $\begin{array}{l}2 \\
8 \\
2 \\
8 \\
8\end{array}$ & $\begin{array}{r}14 \\
4 \\
5 \\
12 \\
8\end{array}$ & $\begin{array}{r}8 \\
15 \\
8 \\
11 \\
13\end{array}$ & $\begin{array}{l}1 \\
7 \\
2 \\
3 \\
5\end{array}$ & $\begin{array}{l}181 \\
182 \\
183 \\
184 \\
185\end{array}$ & $\begin{array}{l}2 \\
0 \\
2 \\
0 \\
0\end{array}$ & $\begin{array}{l}2 \\
0 \\
0 \\
0 \\
4\end{array}$ & $\begin{array}{l}182 \\
336 \\
248 \\
288 \\
228\end{array}$ & $\begin{array}{l}2 \\
8 \\
4 \\
8 \\
4\end{array}$ & $\begin{array}{r}10 \\
12 \\
8 \\
8 \\
16\end{array}$ & $\begin{array}{l}14 \\
25 \\
19 \\
21 \\
17\end{array}$ & $\begin{array}{r}5 \\
10 \\
6 \\
9 \\
5\end{array}$ \\
\hline $\begin{array}{l}106 \\
107 \\
108 \\
109 \\
110\end{array}$ & $\begin{array}{l}0 \\
0 \\
0 \\
2 \\
0\end{array}$ & $\begin{array}{l}2 \\
0 \\
0 \\
2 \\
0\end{array}$ & $\begin{array}{l}162 \\
108 \\
216 \\
110 \\
216\end{array}$ & $\begin{array}{r}4 \\
2 \\
18 \\
2 \\
8\end{array}$ & $\begin{array}{r}6 \\
9 \\
6 \\
6 \\
12\end{array}$ & $\begin{array}{r}12 \\
9 \\
10 \\
8 \\
15\end{array}$ & $\begin{array}{l}5 \\
2 \\
4 \\
3 \\
5\end{array}$ & $\begin{array}{l}186 \\
187 \\
188 \\
189 \\
190\end{array}$ & $\begin{array}{l}0 \\
0 \\
0 \\
0 \\
0\end{array}$ & $\begin{array}{l}0 \\
0 \\
0 \\
0 \\
0\end{array}$ & $\begin{array}{l}384 \\
亡 16 \\
288 \\
288 \\
360\end{array}$ & $\begin{array}{r}8 \\
4 \\
6 \\
12 \\
8\end{array}$ & $\begin{array}{r}12 \\
6 \\
10 \\
12 \\
4\end{array}$ & $\begin{array}{l}29 \\
17 \\
22 \\
19 \\
27\end{array}$ & $\begin{array}{r}12 \\
7 \\
9 \\
7 \\
13\end{array}$ \\
\hline $\begin{array}{l}111 \\
112 \\
113 \\
114 \\
115\end{array}$ & $\begin{array}{l}2 \\
0 \\
0 \\
0 \\
0\end{array}$ & $\begin{array}{l}0 \\
0 \\
2 \\
0 \\
0\end{array}$ & $\begin{array}{l}152 \\
192 \\
114 \\
240 \\
144\end{array}$ & $\begin{array}{r}4 \\
12 \\
2 \\
8 \\
4\end{array}$ & $\begin{array}{l}8 \\
4 \\
8 \\
8 \\
6\end{array}$ & $\begin{array}{r}11 \\
11 \\
9 \\
17 \\
11\end{array}$ & $\begin{array}{l}2 \\
5 \\
3 \\
7 \\
4\end{array}$ & $\begin{array}{l}191 \\
192 \\
193 \\
194 \\
195\end{array}$ & $\begin{array}{l}0 \\
0 \\
2 \\
0 \\
0\end{array}$ & $\begin{array}{l}0 \\
0 \\
2 \\
2 \\
0\end{array}$ & $\begin{array}{l}192 \\
384 \\
194 \\
294 \\
336\end{array}$ & $\begin{array}{r}2 \\
24 \\
2 \\
4 \\
8\end{array}$ & $\begin{array}{r}13 \\
8 \\
4 \\
20 \\
12\end{array}$ & $\begin{array}{l}16 \\
21 \\
15 \\
23 \\
25\end{array}$ & $\begin{array}{l}2 \\
9 \\
7 \\
7 \\
9\end{array}$ \\
\hline $\begin{array}{l}116 \\
117 \\
118 \\
119 \\
120\end{array}$ & $\begin{array}{l}0 \\
0 \\
0 \\
0 \\
0\end{array}$ & $\begin{array}{l}0 \\
0 \\
0 \\
0 \\
0\end{array}$ & $\begin{array}{l}180 \\
168 \\
180 \\
144 \\
288\end{array}$ & $\begin{array}{r}6 \\
8 \\
4 \\
4 \\
16\end{array}$ & $\begin{array}{r}12 \\
8 \\
6 \\
10 \\
8\end{array}$ & $\begin{array}{l}13 \\
11 \\
14 \\
11 \\
17\end{array}$ & $\begin{array}{l}4 \\
4 \\
6 \\
1 \\
7\end{array}$ & $\begin{array}{l}196 \\
197 \\
198 \\
199 \\
200\end{array}$ & $\begin{array}{l}0 \\
0 \\
0 \\
2 \\
0\end{array}$ & $\begin{array}{l}0 \\
2 \\
0 \\
0 \\
0\end{array}$ & $\begin{array}{l}336 \\
198 \\
432 \\
200 \\
360\end{array}$ & $\begin{array}{r}24 \\
2 \\
16 \\
2 \\
24\end{array}$ & $\begin{array}{r}8 \\
10 \\
8 \\
9 \\
12\end{array}$ & $\begin{array}{l}17 \\
16 \\
29 \\
16 \\
19\end{array}$ & $\begin{array}{r}7 \\
6 \\
13 \\
4 \\
7\end{array}$ \\
\hline $\begin{array}{l}121 \\
122 \\
123 \\
124 \\
125\end{array}$ & $\begin{array}{l}0 \\
0 \\
0 \\
0 \\
0\end{array}$ & $\begin{array}{l}0 \\
2 \\
0 \\
0 \\
2\end{array}$ & $\begin{array}{l}132 \\
186 \\
168 \\
192 \\
150\end{array}$ & $\begin{array}{r}12 \\
4 \\
4 \\
6 \\
10\end{array}$ & $\begin{array}{r}6 \\
10 \\
6 \\
6 \\
10\end{array}$ & $\begin{array}{r}6 \\
14 \\
13 \\
14 \\
8\end{array}$ & $\begin{array}{l}2 \\
5 \\
5 \\
6 \\
2\end{array}$ & $\begin{array}{l}201 \\
202 \\
203 \\
204 \\
205\end{array}$ & $\begin{array}{l}2 \\
0 \\
0 \\
0 \\
0\end{array}$ & $\begin{array}{l}0 \\
2 \\
0 \\
0 \\
4\end{array}$ & $\begin{array}{l}272 \\
306 \\
240 \\
432 \\
252\end{array}$ & $\begin{array}{r}4 \\
4 \\
4 \\
12 \\
4\end{array}$ & $\begin{array}{r}12 \\
6 \\
12 \\
12 \\
8\end{array}$ & $\begin{array}{l}21 \\
24 \\
19 \\
31 \\
19\end{array}$ & $\begin{array}{r}8 \\
11 \\
6 \\
13 \\
8\end{array}$ \\
\hline $\begin{array}{l}126 \\
127 \\
128 \\
129 \\
130\end{array}$ & $\begin{array}{l}0 \\
2 \\
0 \\
2 \\
0\end{array}$ & $\begin{array}{l}0 \\
0 \\
0 \\
0 \\
4\end{array}$ & $\begin{array}{l}288 \\
128 \\
192 \\
176 \\
252\end{array}$ & $\begin{array}{r}16 \\
2 \\
16 \\
4 \\
8\end{array}$ & $\begin{array}{r}8 \\
5 \\
8 \\
12 \\
4\end{array}$ & $\begin{array}{r}17 \\
10 \\
9 \\
13 \\
17\end{array}$ & $\begin{array}{l}7 \\
3 \\
3 \\
4 \\
8\end{array}$ & $\begin{array}{l}206 \\
207 \\
208 \\
209 \\
210\end{array}$ & $\begin{array}{l}0 \\
0 \\
0 \\
0 \\
0\end{array}$ & $\begin{array}{l}0 \\
0 \\
0 \\
0 \\
0\end{array}$ & $\begin{array}{l}312 \\
288 \\
336 \\
240 \\
576\end{array}$ & $\begin{array}{r}4 \\
8 \\
12 \\
4 \\
16\end{array}$ & $\begin{array}{r}20 \\
6 \\
8 \\
20 \\
8\end{array}$ & $\begin{array}{l}25 \\
21 \\
23 \\
19 \\
41\end{array}$ & $\begin{array}{r}8 \\
8 \\
10 \\
5 \\
19\end{array}$ \\
\hline $\begin{array}{l}131 \\
132 \\
133 \\
134 \\
135\end{array}$ & $\begin{array}{l}0 \\
0 \\
4 \\
0 \\
0\end{array}$ & $\begin{array}{l}0 \\
0 \\
0 \\
0 \\
0\end{array}$ & $\begin{array}{l}132 \\
288 \\
160 \\
204 \\
216\end{array}$ & $\begin{array}{r}2 \\
12 \\
4 \\
4 \\
12\end{array}$ & $\begin{array}{r}15 \\
8 \\
4 \\
14 \\
6\end{array}$ & $\begin{array}{l}11 \\
19 \\
11 \\
16 \\
13\end{array}$ & $\begin{array}{l}1 \\
8 \\
5 \\
5 \\
4\end{array}$ & $\begin{array}{l}211 \\
212 \\
213 \\
214 \\
215\end{array}$ & $\begin{array}{l}2 \\
0 \\
0 \\
0 \\
0\end{array}$ & $\begin{array}{l}0 \\
0 \\
0 \\
0 \\
0\end{array}$ & $\begin{array}{l}212 \\
324 \\
288 \\
324 \\
264\end{array}$ & $\begin{array}{l}2 \\
6 \\
4 \\
4 \\
4\end{array}$ & $\begin{array}{r}9 \\
12 \\
8 \\
6 \\
14\end{array}$ & $\begin{array}{l}17 \\
25 \\
23 \\
26 \\
21\end{array}$ & $\begin{array}{r}6 \\
10 \\
10 \\
12 \\
4\end{array}$ \\
\hline
\end{tabular}




\begin{tabular}{|c|c|c|c|c|c|c|c|c|c|c|c|c|c|c|c|}
\hline$n$ & $\nu_{1}(n)$ & $\nu_{2}(n)$ & $\mu(n)$ & $\sigma_{0}(n)$ & $h(-4 n)$ & $g_{0}(n)$ & $g^{*}(n)$ & $n$ & $\nu_{1}(n)$ & $\nu_{2}(n)$ & $\mu(n)$ & $\sigma_{0}(n)$ & $h(-4 n)$ & $g_{0}(n)$ & $g^{*}(n)$ \\
\hline $\begin{array}{l}216 \\
217 \\
218 \\
219 \\
220\end{array}$ & $\begin{array}{l}0 \\
4 \\
0 \\
2 \\
0\end{array}$ & $\begin{array}{l}0 \\
0 \\
2 \\
0 \\
0\end{array}$ & $\begin{array}{l}432 \\
256 \\
330 \\
296 \\
432\end{array}$ & $\begin{array}{r}24 \\
4 \\
4 \\
4 \\
12\end{array}$ & $\begin{array}{r}12 \\
8 \\
10 \\
12 \\
8\end{array}$ & $\begin{array}{l}25 \\
19 \\
26 \\
23 \\
31\end{array}$ & $\begin{array}{r}10 \\
8 \\
11 \\
8 \\
14\end{array}$ & $\begin{array}{l}301 \\
302 \\
303 \\
304 \\
305\end{array}$ & $\begin{array}{l}4 \\
0 \\
0 \\
0 \\
0\end{array}$ & $\begin{array}{l}0 \\
0 \\
0 \\
0 \\
4\end{array}$ & $\begin{array}{l}352 \\
456 \\
408 \\
480 \\
372\end{array}$ & $\begin{array}{r}4 \\
4 \\
4 \\
12 \\
4\end{array}$ & $\begin{array}{r}8 \\
12 \\
10 \\
12 \\
16\end{array}$ & $\begin{array}{l}27 \\
37 \\
33 \\
35 \\
29\end{array}$ & $\begin{array}{l}12 \\
16 \\
12 \\
15 \\
11\end{array}$ \\
\hline $\begin{array}{l}221 \\
2222 \\
223 \\
224 \\
225\end{array}$ & $\begin{array}{l}0 \\
0 \\
2 \\
0 \\
0\end{array}$ & $\begin{array}{l}4 \\
0 \\
0 \\
0 \\
0\end{array}$ & $\begin{array}{l}252 \\
456 \\
224 \\
384 \\
360\end{array}$ & $\begin{array}{r}4 \\
8 \\
2 \\
16 \\
24\end{array}$ & $\begin{array}{c}16 \\
12 \\
7 \\
16 \\
8\end{array}$ & $\begin{array}{l}19 \\
35 \\
18 \\
25 \\
19\end{array}$ & $\begin{array}{r}6 \\
15 \\
6 \\
9 \\
8\end{array}$ & $\begin{array}{l}306 \\
307 \\
308 \\
309 \\
310\end{array}$ & $\begin{array}{l}0 \\
2 \\
0 \\
2 \\
0\end{array}$ & $\begin{array}{l}0 \\
0 \\
0 \\
0 \\
0\end{array}$ & $\begin{array}{l}648 \\
308 \\
576 \\
416 \\
576\end{array}$ & $\begin{array}{r}16 \\
2 \\
12 \\
4 \\
8\end{array}$ & $\begin{array}{c}16 \\
9 \\
16 \\
12 \\
8\end{array}$ & $\begin{array}{l}47 \\
25 \\
43 \\
33 \\
45\end{array}$ & $\begin{array}{l}20 \\
10 \\
18 \\
14 \\
21\end{array}$ \\
\hline $\begin{array}{l}226 \\
227 \\
228 \\
229 \\
230\end{array}$ & $\begin{array}{l}0 \\
0 \\
0 \\
2 \\
0\end{array}$ & $\begin{array}{l}2 \\
0 \\
0 \\
2 \\
0\end{array}$ & $\begin{array}{l}342 \\
228 \\
480 \\
230 \\
432\end{array}$ & $\begin{array}{r}4 \\
2 \\
12 \\
2 \\
8\end{array}$ & $\begin{array}{r}8 \\
15 \\
8 \\
10 \\
20\end{array}$ & $\begin{array}{l}27 \\
19 \\
35 \\
18 \\
33\end{array}$ & $\begin{array}{r}12 \\
5 \\
16 \\
7 \\
12\end{array}$ & $\begin{array}{l}311 \\
312 \\
313 \\
314 \\
315\end{array}$ & $\begin{array}{l}0 \\
0 \\
2 \\
0 \\
0\end{array}$ & $\begin{array}{l}0 \\
0 \\
2 \\
2 \\
0\end{array}$ & $\begin{array}{l}312 \\
672 \\
314 \\
474 \\
576\end{array}$ & $\begin{array}{r}2 \\
16 \\
2 \\
4 \\
16\end{array}$ & $\begin{array}{r}19 \\
8 \\
8 \\
26 \\
12\end{array}$ & $\begin{array}{l}26 \\
49 \\
25 \\
38 \\
41\end{array}$ & $\begin{array}{r}4 \\
23 \\
11 \\
13 \\
17\end{array}$ \\
\hline $\begin{array}{l}231 \\
232 \\
233 \\
234 \\
235\end{array}$ & $\begin{array}{l}0 \\
0 \\
0 \\
0 \\
0 \\
0\end{array}$ & $\begin{array}{l}0 \\
0 \\
2 \\
0 \\
0\end{array}$ & $\begin{array}{l}384 \\
360 \\
234 \\
504 \\
288\end{array}$ & $\begin{array}{r}8 \\
8 \\
2 \\
16 \\
4\end{array}$ & $\begin{array}{r}12 \\
4 \\
12 \\
12 \\
6\end{array}$ & $\begin{array}{l}29 \\
27 \\
19 \\
35 \\
23\end{array}$ & $\begin{array}{r}9 \\
13 \\
7 \\
15 \\
10\end{array}$ & $\begin{array}{l}316 \\
317 \\
318 \\
319 \\
320\end{array}$ & $\begin{array}{l}0 \\
0 \\
0 \\
0 \\
0\end{array}$ & $\begin{array}{l}0 \\
2 \\
0 \\
0 \\
0\end{array}$ & $\begin{array}{l}480 \\
318 \\
648 \\
360 \\
576\end{array}$ & $\begin{array}{r}6 \\
2 \\
8 \\
4 \\
24\end{array}$ & $\begin{array}{l}10 \\
10 \\
12 \\
10 \\
16\end{array}$ & $\begin{array}{l}38 \\
26 \\
51 \\
29 \\
37\end{array}$ & $\begin{array}{l}17 \\
11 \\
23 \\
10 \\
15\end{array}$ \\
\hline $\begin{array}{l}236 \\
237 \\
238 \\
239 \\
240\end{array}$ & $\begin{array}{l}0 \\
2 \\
0 \\
0 \\
0\end{array}$ & $\begin{array}{l}0 \\
0 \\
0 \\
0 \\
0\end{array}$ & $\begin{array}{l}360 \\
320 \\
432 \\
240 \\
576\end{array}$ & $\begin{array}{r}6 \\
4 \\
8 \\
2 \\
24\end{array}$ & $\begin{array}{r}18 \\
12 \\
8 \\
15 \\
8\end{array}$ & $\begin{array}{l}28 \\
25 \\
33 \\
20 \\
37\end{array}$ & $\begin{array}{r}10 \\
10 \\
15 \\
3 \\
17\end{array}$ & $\begin{array}{l}321 \\
322 \\
323 \\
324 \\
325\end{array}$ & $\begin{array}{l}0 \\
0 \\
0 \\
0 \\
0\end{array}$ & $\begin{array}{l}0 \\
0 \\
0 \\
0 \\
4\end{array}$ & $\begin{array}{l}432 \\
576 \\
360 \\
648 \\
420\end{array}$ & $\begin{array}{r}4 \\
8 \\
4 \\
36 \\
12\end{array}$ & $\begin{array}{c}20 \\
8 \\
12 \\
12 \\
12\end{array}$ & $\begin{array}{l}35 \\
45 \\
29 \\
37 \\
29\end{array}$ & $\begin{array}{l}13 \\
21 \\
11 \\
16 \\
12\end{array}$ \\
\hline $\begin{array}{l}241 \\
242 \\
243 \\
244 \\
245\end{array}$ & $\begin{array}{l}2 \\
0 \\
0 \\
0 \\
0\end{array}$ & $\begin{array}{l}2 \\
0 \\
0 \\
0 \\
0\end{array}$ & $\begin{array}{l}242 \\
396 \\
324 \\
372 \\
336\end{array}$ & $\begin{array}{r}2 \\
24 \\
18 \\
6 \\
16\end{array}$ & $\begin{array}{r}12 \\
10 \\
9 \\
12 \\
12\end{array}$ & $\begin{array}{l}19 \\
22 \\
19 \\
29 \\
21\end{array}$ & $\begin{array}{r}7 \\
9 \\
7 \\
12 \\
8\end{array}$ & $\begin{array}{l}326 \\
327 \\
328 \\
329 \\
330\end{array}$ & $\begin{array}{l}0 \\
2 \\
0 \\
0 \\
0\end{array}$ & $\begin{array}{l}0 \\
0 \\
0 \\
0 \\
0\end{array}$ & $\begin{array}{l}492 \\
440 \\
504 \\
384 \\
864\end{array}$ & $\begin{array}{r}4 \\
4 \\
8 \\
4 \\
16\end{array}$ & $\begin{array}{r}22 \\
12 \\
8 \\
24 \\
8\end{array}$ & $\begin{array}{l}40 \\
35 \\
39 \\
31 \\
65\end{array}$ & $\begin{array}{l}15 \\
12 \\
18 \\
10 \\
31\end{array}$ \\
\hline $\begin{array}{l}246 \\
247 \\
248 \\
249 \\
250\end{array}$ & $\begin{array}{l}0 \\
4 \\
0 \\
0 \\
0\end{array}$ & $\begin{array}{l}0 \\
0 \\
0 \\
0 \\
2\end{array}$ & $\begin{array}{l}504 \\
280 \\
384 \\
336 \\
450\end{array}$ & $\begin{array}{r}8 \\
4 \\
8 \\
4 \\
\end{array}$ & $\begin{array}{r}12 \\
6 \\
16 \\
12 \\
10\end{array}$ & $\begin{array}{l}39 \\
21 \\
29 \\
27 \\
28\end{array}$ & $\begin{array}{r}17 \\
8 \\
11 \\
11 \\
11\end{array}$ & $\begin{array}{l}331 \\
332 \\
333 \\
334 \\
335\end{array}$ & $\begin{array}{l}2 \\
0 \\
0 \\
0 \\
0\end{array}$ & $\begin{array}{l}0 \\
0 \\
0 \\
0 \\
0\end{array}$ & $\begin{array}{l}332 \\
504 \\
456 \\
504 \\
408\end{array}$ & $\begin{array}{l}2 \\
6 \\
8 \\
4 \\
4\end{array}$ & $\begin{array}{r}9 \\
18 \\
8 \\
12 \\
18\end{array}$ & $\begin{array}{l}27 \\
40 \\
35 \\
41 \\
33\end{array}$ & $\begin{array}{l}11 \\
16 \\
16 \\
18 \\
8\end{array}$ \\
\hline $\begin{array}{l}251 \\
252 \\
253 \\
254 \\
255\end{array}$ & $\begin{array}{l}0 \\
0 \\
0 \\
0 \\
0\end{array}$ & $\begin{array}{l}0 \\
0 \\
0 \\
0 \\
0\end{array}$ & $\begin{array}{l}252 \\
576 \\
288 \\
384 \\
432\end{array}$ & $\begin{array}{r}2 \\
24 \\
4 \\
4 \\
8\end{array}$ & $\begin{array}{r}21 \\
8 \\
4 \\
16 \\
12\end{array}$ & $\begin{array}{l}21 \\
37 \\
23 \\
31 \\
33\end{array}$ & $\begin{array}{l}4 \\
17 \\
11 \\
12 \\
11\end{array}$ & $\begin{array}{l}336 \\
337 \\
338 \\
339 \\
340\end{array}$ & $\begin{array}{l}0 \\
2 \\
0 \\
0 \\
0\end{array}$ & $\begin{array}{l}0 \\
2 \\
2 \\
0 \\
0\end{array}$ & $\begin{array}{l}768 \\
338 \\
546 \\
456 \\
648\end{array}$ & $\begin{array}{c}24 \\
2 \\
28 \\
4 \\
12\end{array}$ & $\begin{array}{r}16 \\
8 \\
14 \\
18 \\
8\end{array}$ & $\begin{array}{l}53 \\
27 \\
32 \\
37 \\
49\end{array}$ & $\begin{array}{l}23 \\
12 \\
13 \\
13 \\
23\end{array}$ \\
\hline $\begin{array}{l}256 \\
257 \\
258 \\
259 \\
260\end{array}$ & $\begin{array}{l}0 \\
0 \\
0 \\
4 \\
0\end{array}$ & $\begin{array}{l}0 \\
2 \\
0 \\
0 \\
0\end{array}$ & $\begin{array}{l}384 \\
258 \\
528 \\
304 \\
504\end{array}$ & $\begin{array}{r}24 \\
2 \\
8 \\
4 \\
12\end{array}$ & $\begin{array}{r}8 \\
16 \\
8 \\
12 \\
16\end{array}$ & $\begin{array}{l}21 \\
21 \\
41 \\
23 \\
37\end{array}$ & $\begin{array}{r}9 \\
7 \\
19 \\
8 \\
15\end{array}$ & $\begin{array}{l}341 \\
342 \\
343 \\
344 \\
345\end{array}$ & $\begin{array}{l}0 \\
0 \\
2 \\
0 \\
0\end{array}$ & $\begin{array}{l}0 \\
0 \\
0 \\
0 \\
0\end{array}$ & $\begin{array}{l}384 \\
720 \\
392 \\
528 \\
576\end{array}$ & $\begin{array}{r}4 \\
16 \\
14 \\
8 \\
8\end{array}$ & $\begin{array}{r}28 \\
12 \\
70 \\
20 \\
8\end{array}$ & $\begin{array}{l}31 \\
53 \\
26 \\
41 \\
45\end{array}$ & $\begin{array}{r}9 \\
24 \\
10 \\
16 \\
21\end{array}$ \\
\hline $\begin{array}{l}261 \\
262 \\
263 \\
264 \\
265\end{array}$ & $\begin{array}{l}0 \\
0 \\
0 \\
0 \\
0\end{array}$ & $\begin{array}{l}0 \\
0 \\
0 \\
0 \\
4\end{array}$ & $\begin{array}{l}360 \\
396 \\
264 \\
576 \\
324\end{array}$ & $\begin{array}{r}8 \\
4 \\
2 \\
16 \\
4\end{array}$ & $\begin{array}{c}12 \\
6 \\
13 \\
16 \\
8\end{array}$ & $\begin{array}{l}27 \\
32 \\
22 \\
41 \\
25\end{array}$ & $\begin{array}{r}11 \\
15 \\
5 \\
17 \\
11\end{array}$ & $\begin{array}{l}346 \\
347 \\
348 \\
349 \\
350\end{array}$ & $\begin{array}{l}0 \\
0 \\
0 \\
2 \\
0\end{array}$ & $\begin{array}{l}2 \\
0 \\
0 \\
2 \\
0\end{array}$ & $\begin{array}{l}522 \\
348 \\
720 \\
350 \\
720\end{array}$ & $\begin{array}{r}4 \\
2 \\
12 \\
2 \\
24\end{array}$ & $\begin{array}{l}10 \\
15 \\
12 \\
14 \\
16\end{array}$ & $\begin{array}{l}42 \\
29 \\
55 \\
28 \\
49\end{array}$ & $\begin{array}{l}19 \\
10 \\
25 \\
11 \\
21\end{array}$ \\
\hline $\begin{array}{l}266 \\
267 \\
268 \\
269 \\
270\end{array}$ & $\begin{array}{l}0 \\
0 \\
0 \\
0 \\
0\end{array}$ & $\begin{array}{l}0 \\
0 \\
0 \\
2 \\
0\end{array}$ & $\begin{array}{l}480 \\
360 \\
408 \\
270 \\
648\end{array}$ & $\begin{array}{r}8 \\
4 \\
6 \\
2 \\
24\end{array}$ & $\begin{array}{r}20 \\
6 \\
6 \\
22 \\
12\end{array}$ & $\begin{array}{l}37 \\
29 \\
32 \\
22 \\
43\end{array}$ & $\begin{array}{r}14 \\
13 \\
15 \\
6 \\
19\end{array}$ & $\begin{array}{l}351 \\
352 \\
353 \\
354 \\
355\end{array}$ & $\begin{array}{l}0 \\
0 \\
0 \\
0 \\
0\end{array}$ & $\begin{array}{l}0 \\
0 \\
2 \\
0 \\
0\end{array}$ & $\begin{array}{l}504 \\
576 \\
354 \\
720 \\
432\end{array}$ & $\begin{array}{r}12 \\
16 \\
2 \\
8 \\
4\end{array}$ & $\begin{array}{l}12 \\
8 \\
16 \\
16 \\
12\end{array}$ & $\begin{array}{l}37 \\
41 \\
29 \\
57 \\
35\end{array}$ & $\begin{array}{l}13 \\
19 \\
11 \\
25 \\
14\end{array}$ \\
\hline $\begin{array}{l}271 \\
272 \\
273 \\
273 \\
274 \\
275\end{array}$ & $\begin{array}{l}2 \\
0 \\
4 \\
0 \\
0\end{array}$ & $\begin{array}{l}0 \\
0 \\
0 \\
2 \\
0\end{array}$ & $\begin{array}{l}272 \\
432 \\
448 \\
414 \\
360\end{array}$ & $\begin{array}{r}2 \\
12 \\
8 \\
4 \\
12\end{array}$ & $\begin{array}{r}11 \\
16 \\
8 \\
12 \\
12\end{array}$ & $\begin{array}{l}22 \\
31 \\
33 \\
33 \\
25\end{array}$ & $\begin{array}{r}6 \\
12 \\
15 \\
14 \\
9\end{array}$ & $\begin{array}{l}356 \\
357 \\
358 \\
359 \\
360\end{array}$ & $\begin{array}{l}0 \\
0 \\
0 \\
0 \\
0\end{array}$ & $\begin{array}{l}0 \\
0 \\
0 \\
0 \\
0\end{array}$ & $\begin{array}{l}540 \\
576 \\
540 \\
360 \\
864\end{array}$ & $\begin{array}{r}6 \\
8 \\
4 \\
2 \\
32\end{array}$ & $\begin{array}{r}24 \\
8 \\
6 \\
19 \\
16\end{array}$ & $\begin{array}{l}43 \\
45 \\
44 \\
30 \\
57\end{array}$ & $\begin{array}{l}16 \\
21 \\
21 \\
6 \\
25\end{array}$ \\
\hline $\begin{array}{l}276 \\
277 \\
278 \\
279 \\
280\end{array}$ & $\begin{array}{l}0 \\
2 \\
0 \\
0 \\
0\end{array}$ & $\begin{array}{l}0 \\
2 \\
0 \\
0 \\
0\end{array}$ & $\begin{array}{l}576 \\
278 \\
420 \\
384 \\
576\end{array}$ & $\begin{array}{r}12 \\
2 \\
4 \\
8 \\
16\end{array}$ & $\begin{array}{c}16 \\
6 \\
14 \\
12 \\
8\end{array}$ & $\begin{array}{l}43 \\
22 \\
34 \\
29 \\
41\end{array}$ & $\begin{array}{l}18 \\
10 \\
14 \\
9 \\
19\end{array}$ & $\begin{array}{l}361 \\
362 \\
363 \\
364 \\
365\end{array}$ & $\begin{array}{l}2 \\
0 \\
0 \\
0 \\
0\end{array}$ & $\begin{array}{l}0 \\
2 \\
0 \\
0 \\
4\end{array}$ & $\begin{array}{l}380 \\
546 \\
528 \\
672 \\
444\end{array}$ & $\begin{array}{r}20 \\
4 \\
24 \\
12 \\
4\end{array}$ & $\begin{array}{l}10 \\
18 \\
12 \\
12 \\
20\end{array}$ & $\begin{array}{l}22 \\
44 \\
33 \\
51 \\
35\end{array}$ & $\begin{array}{l}9 \\
18 \\
13 \\
23 \\
13\end{array}$ \\
\hline $\begin{array}{l}281 \\
282 \\
283 \\
284 \\
285\end{array}$ & $\begin{array}{l}0 \\
0 \\
2 \\
0 \\
0\end{array}$ & $\begin{array}{l}2 \\
0 \\
0 \\
0 \\
0\end{array}$ & $\begin{array}{l}282 \\
576 \\
284 \\
432 \\
480\end{array}$ & $\begin{array}{l}2 \\
8 \\
2 \\
6 \\
8\end{array}$ & $\begin{array}{r}20 \\
8 \\
9 \\
14 \\
16\end{array}$ & $\begin{array}{l}23 \\
45 \\
23 \\
34 \\
37\end{array}$ & $\begin{array}{r}7 \\
21 \\
9 \\
14 \\
15\end{array}$ & $\begin{array}{l}366 \\
367 \\
368 \\
369 \\
370\end{array}$ & $\begin{array}{l}0 \\
2 \\
0 \\
0 \\
0\end{array}$ & $\begin{array}{l}0 \\
0 \\
0 \\
0 \\
4\end{array}$ & $\begin{array}{l}744 \\
368 \\
576 \\
504 \\
684\end{array}$ & $\begin{array}{r}8 \\
2 \\
12 \\
8 \\
8\end{array}$ & $\begin{array}{l}12 \\
9 \\
12 \\
16 \\
12\end{array}$ & $\begin{array}{l}59 \\
30 \\
43 \\
39 \\
53\end{array}$ & $\begin{array}{l}27 \\
11 \\
19 \\
16 \\
24\end{array}$ \\
\hline $\begin{array}{l}286 \\
287 \\
288 \\
289 \\
290\end{array}$ & $\begin{array}{l}0 \\
0 \\
0 \\
0 \\
0\end{array}$ & $\begin{array}{l}0 \\
0 \\
0 \\
2 \\
4\end{array}$ & $\begin{array}{l}504 \\
336 \\
576 \\
306 \\
540\end{array}$ & $\begin{array}{r}8 \\
4 \\
32 \\
18 \\
8\end{array}$ & $\begin{array}{r}12 \\
14 \\
8 \\
8 \\
20\end{array}$ & $\begin{array}{l}39 \\
27 \\
33 \\
17 \\
41\end{array}$ & $\begin{array}{c}17 \\
7 \\
15 \\
7 \\
16\end{array}$ & $\begin{array}{l}371 \\
372 \\
373 \\
374 \\
375\end{array}$ & $\begin{array}{l}0 \\
0 \\
2 \\
0 \\
0\end{array}$ & $\begin{array}{l}0 \\
0 \\
2 \\
0 \\
0\end{array}$ & $\begin{array}{l}432 \\
768 \\
374 \\
648 \\
600\end{array}$ & $\begin{array}{r}4 \\
12 \\
2 \\
8 \\
20\end{array}$ & $\begin{array}{r}24 \\
8 \\
10 \\
28 \\
10\end{array}$ & $\begin{array}{l}35 \\
59 \\
30 \\
51 \\
41\end{array}$ & $\begin{array}{l}10 \\
28 \\
13 \\
19 \\
16\end{array}$ \\
\hline $\begin{array}{l}291 \\
292 \\
293 \\
294 \\
295\end{array}$ & $\begin{array}{l}2 \\
0 \\
0 \\
0 \\
0\end{array}$ & $\begin{array}{l}0 \\
0 \\
2 \\
0 \\
0\end{array}$ & $\begin{array}{l}392 \\
444 \\
294 \\
672 \\
360\end{array}$ & $\begin{array}{r}4 \\
6 \\
2 \\
32 \\
4 \\
4\end{array}$ & $\begin{array}{r}12 \\
8 \\
18 \\
12 \\
8\end{array}$ & $\begin{array}{l}31 \\
35 \\
24 \\
41 \\
29\end{array}$ & $\begin{array}{l}12 \\
16 \\
8 \\
18 \\
11\end{array}$ & $\begin{array}{l}376 \\
377 \\
378 \\
379 \\
380\end{array}$ & $\begin{array}{l}0 \\
0 \\
0 \\
2 \\
0\end{array}$ & $\begin{array}{l}0 \\
4 \\
0 \\
0 \\
0\end{array}$ & $\begin{array}{l}576 \\
420 \\
864 \\
380 \\
720\end{array}$ & $\begin{array}{r}8 \\
4 \\
24 \\
2 \\
12\end{array}$ & $\begin{array}{c}16 \\
16 \\
12 \\
9 \\
16\end{array}$ & $\begin{array}{l}45 \\
33 \\
61 \\
31 \\
55\end{array}$ & $\begin{array}{l}19 \\
13 \\
28 \\
13 \\
24\end{array}$ \\
\hline $\begin{array}{l}296 \\
297 \\
298 \\
299 \\
300\end{array}$ & $\begin{array}{l}0 \\
0 \\
0 \\
0 \\
0\end{array}$ & $\begin{array}{l}0 \\
0 \\
2 \\
0 \\
0\end{array}$ & $\begin{array}{l}456 \\
432 \\
450 \\
336 \\
720\end{array}$ & $\begin{array}{r}8 \\
12 \\
4 \\
4 \\
36\end{array}$ & $\begin{array}{l}20 \\
12 \\
6 \\
24 \\
12\end{array}$ & $\begin{array}{l}35 \\
31 \\
36 \\
27 \\
43\end{array}$ & $\begin{array}{c}13 \\
13 \\
17 \\
6 \\
19\end{array}$ & $\begin{array}{l}381 \\
382 \\
383 \\
384 \\
385\end{array}$ & $\begin{array}{l}2 \\
0 \\
0 \\
0 \\
0\end{array}$ & $\begin{array}{l}0 \\
0 \\
0 \\
0 \\
0\end{array}$ & $\begin{array}{l}512 \\
576 \\
384 \\
768 \\
576\end{array}$ & $\begin{array}{r}4 \\
4 \\
2 \\
32 \\
8\end{array}$ & $\begin{array}{r}20 \\
8 \\
17 \\
16 \\
8\end{array}$ & $\begin{array}{l}41 \\
47 \\
32 \\
49 \\
45\end{array}$ & $\begin{array}{l}16 \\
22 \\
8 \\
21 \\
21\end{array}$ \\
\hline
\end{tabular}




\begin{tabular}{c|c|c|c|c|c|c|c|}
\hline \hline$n$ & $\nu_{1}(n)$ & $\nu_{2}(n)$ & $\mu(n)$ & $\sigma_{0}(n)$ & $h(-4 n)$ & $g_{0}(n)$ & $g^{*}(n)$ \\
\cline { 2 - 6 } & 0 & 2 & 582 & 4 & 20 & 47 & 19 \\
\hline 386 & 0 & 0 & 528 & 8 & 12 & 41 & 17 \\
\hline 388 & & & &
\end{tabular}

\begin{tabular}{|c|c|c|c|c|c|c|c|}
\hline$n$ & $\nu_{1}(n)$ & $\nu_{2}(n)$ & $\mu(n)$ & $\sigma_{0}(n)$ & $h(-4 n)$ & $g_{0}(n)$ & $g^{*}(n)$ \\
\hline 471 & 2 & 0 & 632 & 4 & 16 & 51 & 18 \\
\hline 472 & 0 & 0 & 720 & $\begin{array}{l}7 \\
8\end{array}$ & $\begin{array}{l}10 \\
12\end{array}$ & $\begin{array}{l}51 \\
57\end{array}$ & $\begin{array}{l}10 \\
26\end{array}$ \\
\hline 473 & 0 & 0 & 528 & 4 & 12 & 43 & 19 \\
\hline $\begin{array}{l}470 \\
474\end{array}$ & 0 & 0 & $\begin{array}{l}328 \\
960\end{array}$ & $\begin{array}{l}4 \\
8\end{array}$ & $\begin{array}{l}12 \\
20\end{array}$ & $\begin{array}{l}43 \\
77\end{array}$ & $\begin{array}{l}19 \\
34\end{array}$ \\
\hline 475 & 0 & 0 & 600 & 12 & 12 & 45 & 19 \\
\hline 476 & 0 & 0 & 864 & 12 & 20 & 67 & 29 \\
\hline
\end{tabular}

\begin{tabular}{l|l|l|l|r|r|r|l}
391 & 0 & 0 & 432 & 4 & 14 & 35 & 11 \\
392 & 0 & 0 & 672 & 32 & 16 & 41 & 17 \\
393 & 0 & 0 & 528 & 4 & 12 & 43 & 19 \\
394 & 0 & 2 & 594 & 4 & 10 & 48 & 22 \\
395 & 0 & 0 & 480 & 4 & 24 & 39 & 12 \\
396 & 0 & 0 & 864 & 24 & 12 & 61 & 28 \\
397 & 2 & 2 & 398 & 2 & 6 & 32 & 15 \\
398 & 0 & 0 & 600 & 4 & 20 & 49 & 20 \\
399 & 4 & 0 & 640 & 8 & 16 & 49 & 17 \\
400 & 0 & 0 & 720 & 36 & 8 & 43 & 20
\end{tabular}

401

402
403

403
404
405

406

406
408
409

408
409
410

411

412
413
414

415

416
417
418
419
420

421

422
423
424

425

426
427

428
429
4

430

\begin{tabular}{l|l|l|l|r|r|r|r}
431 & 0 & 0 & 432 & 2 & 21 & 36 & 8 \\
432 & 0 & 0 & 864 & 36 & 12 & 55 & 25 \\
433 & 2 & 2 & 434 & 2 & 12 & 35 & 15 \\
434 & 0 & 0 & 768 & 8 & 24 & 61 & 25
\end{tabular}

435

4

43
43
44

4

44
44
44

443
444

440
44

448

45

45

45
45
45

45
45
45
45
460

\begin{tabular}{r|r|r|r|r|r|r|r} 
& & & & & & & \\
& 2 & 2 & 458 & 2 & 8 & 3 & 33 \\
458 & 0 & 2 & 690 & 4 & 26 & 56 & 17 \\
459 & 0 & 0 & 648 & 12 & 18 & 49 & 19
\end{tabular}

\begin{tabular}{l|l|l|r|r|r|r|r}
461 & 0 & 2 & 462 & 2 & 30 & 38 & 12 \\
462 & 0 & 0 & 1152 & 16 & 8 & 89 & 43 \\
463 & 2 & 0 & 464 & 2 & 7 & 38 & 16 \\
464 & 0 & 0 & 720 & 12 & 24 & 55 & 22 \\
465 & 0 & 0 & 768 & 8 & 16 & 61 & 27
\end{tabular}

\begin{tabular}{l|l|l|r|r|r|r|r}
461 & 0 & 2 & 462 & 2 & 30 & 38 & 12 \\
462 & 0 & 0 & 1152 & 16 & 8 & 89 & 43 \\
463 & 2 & 0 & 464 & 2 & 7 & 38 & 16 \\
464 & 0 & 0 & 720 & 12 & 24 & 55 & 22 \\
465 & 0 & 0 & 768 & 8 & 16 & 61 & 27
\end{tabular}

\begin{tabular}{l|l|l|r|r|r|r|r}
466 & 0 & 2 & 702 & 4 & 8 & 57 & 27 \\
467 & 0 & 0 & 468 & 2 & 21 & 39 & 13 \\
468 & 0 & 0 & 1008 & 24 & 16 & 73 & 33 \\
469 & 4 & 0 & 544 & 4 & 16 & 43 & 18 \\
470 & 0 & 0 & 864 & 8 & 20 & 69 & 30
\end{tabular}
476
477
478
479

430

481
482

483

485

486
$4 \times 7$

488
439

490

491

492
493

494
495

496

497
498

499
500

501

502
503

504
505

506

507

508
509

510

511

512
513

514
515

516

517
518

519
520

521

522
523

524
525

526

527
528
529

529
530

531

532

533
534
535

536

537

538
539
540

541

541
542
543

543
544
545

546
547
548

548
549

550

551

553

554 \begin{tabular}{r|r|r|l|l}
\hline 864 & 12 & 20 & 67 & 29 \\
648 & 8 & 12 & 51 & 23 \\
\hline
\end{tabular}

\begin{tabular}{r|r|r|r|r}
720 & 4 & 8 & 59 & 28 \\
480 & 2 & 25 & 40 & 8 \\
1152 & 32 & 16 & 81 & 37
\end{tabular}

\begin{tabular}{l|r|l|l|l}
726 & 4 & 20 & 59 & 25 \\
768 & 8 & 12 & 61 & 27 \\
792 & 36 & 12 & 49 & 22 \\
588 & 4 & 20 & 47 & 19
\end{tabular}

\begin{tabular}{r|r|r|r|r}
972 & 36 & 18 & 64 & 28 \\
488 & 2 & 7 & 40 & 17 \\
744 & 8 & 20 & 59 & 25 \\
656 & 4 & 20 & 53 & 22 \\
1008 & 32 & 12 & 69 & 32
\end{tabular}

\begin{tabular}{r|r|l|l|l}
540 & 4 & 12 & 43 & 37 \\
840 & 8 & 28 & 67 & 19 \\
\hline
\end{tabular}

\begin{tabular}{l|r|l|l|l}
840 & 8 & 28 & 67 & 27 \\
864 & 16 & 16 & 65 & 25 \\
\hline
\end{tabular}

\begin{tabular}{|r|r|l|l|l}
768 & 12 & 12 & 59 & 27 \\
576 & 4 & 24 & 47 & 18 \\
\hline
\end{tabular}

\begin{tabular}{r|r|r|r|r}
1008 & 8 & 8 & 81 & 39 \\
500 & 2 & 9 & 41 & 18
\end{tabular}

\begin{tabular}{l|r|r|r|r}
500 & 2 & 9 & 41 & 18 \\
900 & 30 & 20 & 61 & 26 \\
\hline
\end{tabular}

\begin{tabular}{l|l|l|l|l}
672 & 4 & 16 & 55 & 24 \\
756 & 4 & 14 & 62 & 28 \\
\hline
\end{tabular}

\begin{tabular}{r|r|r|r|r}
612 & 4 & 8 & 49 & 23
\end{tabular}

\begin{tabular}{l|r|l|l|l}
864 & 8 & 28 & 69 & 28 \\
728 & 28 & 12 & 47 & 20
\end{tabular}

\begin{tabular}{r|r|r|r|r}
768 & 6 & 10 & 62 & 29 \\
510 & 2 & 30 & 42 & 14 \\
1296 & 16 & 16 & 101 & 47 \\
\hline
\end{tabular}

\begin{tabular}{r|r|l|l|l}
768 & 32 & 16 & 49 & 21 \\
720 & 12 & 12 & 55 & 25 \\
774 & 4 & 16 & 63 & 28
\end{tabular}

\begin{tabular}{l|l|l|l|l}
774 & 4 & 16 & 63 & 28 \\
624 & 4 & 18 & 51 & 20 \\
\hline
\end{tabular}

\begin{tabular}{l|l|l|l|l}
1056 & 12 & 24 & 83 & 36 \\
\hline
\end{tabular}

\begin{tabular}{|l|l|l|l|l}
576 & 4 & 12 & 47 & 21 \\
912 & 8 & 16 & 73 & 33 \\
\hline
\end{tabular}

\begin{tabular}{r|r|r|r|r}
696 & 4 & 18 & 57 & 33 \\
1008 & 16 & 8 & 77 & 37 \\
\hline
\end{tabular}

\begin{tabular}{r|r|r|l|l}
522 & 2 & 32 & 43 & 14 \\
1080 & 16 & 8 & 83 & 40
\end{tabular}

\begin{tabular}{r|r|r|l|l}
524 & 2 & 15 & 43 & 17 \\
792 & 6 & 30 & 64 & 25 \\
960 & 24 & 16 & 69 & 31
\end{tabular}

\begin{tabular}{r|r|r|r|r}
792 & 4 & 12 & 65 & 30 \\
576 & 4 & 18 & 47 & 15 \\
1152 & 24 & 16 & 85 & 39
\end{tabular}

\begin{tabular}{r|r|l|l|l}
1152 & 24 & 16 & 85 & 39 \\
552 & 24 & 12 & 35 & 15 \\
972 & 8 & 28 & 77
\end{tabular}

\begin{tabular}{r|r|r|r|r}
720 & 8 & 18 & 57 & 23 \\
960 & 12 & 8 & 75 & 36 \\
\hline
\end{tabular}

\begin{tabular}{r|r|r|r|r}
588 & 4 & 8 & 47 & 21 \\
1080 & 8 & 12 & 47 & 3 \\
648 & 4 & 14 & 53 & 20
\end{tabular}

\begin{tabular}{r|l|l|l|l}
1080 & 8 & 20 & 87 & 39 \\
648 & 4 & 14 & 53 & 20
\end{tabular}

\begin{tabular}{l|l|l|l|l}
816 & 8 & 28 & 65 & 26 \\
720 & 4 & 12 & 59 & 27 \\
810 & 4 & 10 & 66
\end{tabular}

\begin{tabular}{r|r|l|l|l}
810 & 4 & 10 & 66 & 31 \\
672 & 16 & 24 & 49 & 17 \\
1296 & 36 & 12 & 91 & 43
\end{tabular}

\begin{tabular}{r|r|r|r|r}
542 & 2 & 10 & 44 & 20 \\
816 & 4 & 24 & 67 & 28 \\
723 & 4 & 12 & 59 & 24 \\
861 & 16 & 16 & 65 & 29 \\
650 & 4 & 32 & 53 & 19 \\
1344 & 16 & 24 & 105 & 47 \\
543 & 2 & 9 & 45 & 20 \\
828 & 6 & 16 & 67 & 30 \\
744 & 8 & 24 & 59 & 24 \\
1080 & 24 & 12 & 79 & 37 \\
600 & 4 & 26 & 49 & 12 \\
1152 & 16 & 16 & 89 & 41 \\
640 & 4 & 8 & 51 & 24 \\
834 & 4 & 22 & 68 & 29 \\
912 & 8 & 12 & 73 & 33
\end{tabular} \begin{tabular}{|l|l|l|l|l}
532 & 4 & 16 & 41 & 17 \\
\hline 798 & 4 & 20 & 59 & 27 \\
\hline
\end{tabular}

\begin{tabular}{r|r|r|r|r}
492 & 2 & 27 & 41 & 12 \\
\hline 1008 & 12 & 12 & 79 & 37 \\
\hline
\end{tabular}

\begin{tabular}{r|r|l|l|l}
504 & 2 & 21 & 42 & 11 \\
1152 & 32 & 16 & 81 & 37
\end{tabular}

\begin{tabular}{|l|l|l|l|l}
592 & 4 & 14 & 47 & 17
\end{tabular}

\begin{tabular}{l|l|l|l|l|}
\hline 1008 & 16 & 8 & 77 & 37 \\
\hline
\end{tabular} 


\begin{tabular}{|c|c|c|c|c|c|c|c|c|c|c|c|c|c|c|c|}
\hline$n$ & $\nu_{1}(n)$ & $\nu_{2}(n)$ & $\mu(n)$ & $\sigma_{0}(n)$ & $h(-4 n)$ & $g_{0}(n)$ & $g^{*}(n)$ & $n$ & $\nu_{1}(n)$ & $\nu_{2}(n)$ & $\mu(n)$ & $\sigma_{0}(n)$ & $h(-4 n)$ & $g_{0}(n)$ & $g^{*}(n)$ \\
\hline $\begin{array}{l}556 \\
557 \\
558 \\
559 \\
560\end{array}$ & $\begin{array}{l}0 \\
0 \\
0 \\
4 \\
0\end{array}$ & $\begin{array}{l}0 \\
2 \\
0 \\
0 \\
0\end{array}$ & $\begin{array}{r}840 \\
558 \\
1152 \\
616 \\
1152\end{array}$ & $\begin{array}{r}6 \\
2 \\
16 \\
4 \\
24\end{array}$ & $\begin{array}{l}18 \\
18 \\
16 \\
16 \\
24\end{array}$ & $\begin{array}{l}68 \\
46 \\
89 \\
49 \\
85\end{array}$ & $\begin{array}{l}30 \\
19 \\
41 \\
17 \\
37\end{array}$ & $\begin{array}{l}641 \\
642 \\
643 \\
644 \\
645\end{array}$ & $\begin{array}{l}0 \\
0 \\
2 \\
0 \\
0\end{array}$ & $\begin{array}{l}2 \\
0 \\
0 \\
0 \\
0\end{array}$ & $\begin{array}{r}642 \\
1296 \\
644 \\
1152 \\
1056\end{array}$ & $\begin{array}{r}2 \\
8 \\
2 \\
12 \\
8\end{array}$ & $\begin{array}{r}28 \\
16 \\
9 \\
32 \\
16\end{array}$ & $\begin{array}{r}53 \\
105 \\
53 \\
91 \\
85\end{array}$ & $\begin{array}{l}20 \\
49 \\
24 \\
38 \\
39\end{array}$ \\
\hline $\begin{array}{l}561 \\
562 \\
563 \\
564 \\
565\end{array}$ & $\begin{array}{l}0 \\
0 \\
0 \\
0 \\
0\end{array}$ & $\begin{array}{l}0 \\
2 \\
0 \\
0 \\
4\end{array}$ & $\begin{array}{r}864 \\
846 \\
564 \\
1152 \\
684\end{array}$ & $\begin{array}{r}8 \\
4 \\
2 \\
12 \\
4\end{array}$ & $\begin{array}{r}16 \\
8 \\
27 \\
16 \\
12\end{array}$ & $\begin{array}{l}69 \\
69 \\
47 \\
91 \\
55\end{array}$ & $\begin{array}{l}31 \\
33 \\
15 \\
42 \\
25\end{array}$ & $\begin{array}{l}646 \\
647 \\
649 \\
649 \\
650\end{array}$ & $\begin{array}{l}0 \\
0 \\
0 \\
0 \\
0\end{array}$ & $\begin{array}{l}0 \\
0 \\
0 \\
0 \\
4\end{array}$ & $\begin{array}{r}1080 \\
648 \\
1296 \\
720 \\
1260\end{array}$ & $\begin{array}{r}8 \\
2 \\
48 \\
4 \\
24\end{array}$ & $\begin{array}{l}16 \\
23 \\
12 \\
20 \\
24\end{array}$ & $\begin{array}{l}87 \\
54 \\
85 \\
59 \\
93\end{array}$ & $\begin{array}{l}40 \\
16 \\
40 \\
25 \\
41\end{array}$ \\
\hline $\begin{array}{l}566 \\
567 \\
568 \\
569 \\
570\end{array}$ & $\begin{array}{l}0 \\
0 \\
0 \\
0 \\
0\end{array}$ & $\begin{array}{l}0 \\
0 \\
0 \\
2 \\
0\end{array}$ & $\begin{array}{r}852 \\
864 \\
864 \\
570 \\
1440\end{array}$ & $\begin{array}{r}4 \\
24 \\
8 \\
2 \\
16\end{array}$ & $\begin{array}{r}30 \\
12 \\
8 \\
32 \\
16\end{array}$ & $\begin{array}{r}70 \\
61 \\
69 \\
47 \\
113\end{array}$ & $\begin{array}{l}28 \\
25 \\
33 \\
16 \\
53\end{array}$ & $\begin{array}{l}651 \\
652 \\
653 \\
654 \\
655\end{array}$ & $\begin{array}{l}4 \\
0 \\
0 \\
0 \\
0\end{array}$ & $\begin{array}{l}0 \\
0 \\
2 \\
0 \\
0\end{array}$ & $\begin{array}{r}1024 \\
984 \\
654 \\
1320 \\
792\end{array}$ & $\begin{array}{l}8 \\
6 \\
2 \\
8 \\
4\end{array}$ & $\begin{array}{r}24 \\
6 \\
14 \\
28 \\
12\end{array}$ & $\begin{array}{r}81 \\
80 \\
54 \\
107 \\
65\end{array}$ & $\begin{array}{l}33 \\
39 \\
24 \\
47 \\
27\end{array}$ \\
\hline $\begin{array}{l}571 \\
572 \\
573 \\
574 \\
575\end{array}$ & $\begin{array}{l}2 \\
0 \\
0 \\
0 \\
0\end{array}$ & $\begin{array}{l}0 \\
0 \\
0 \\
0 \\
0\end{array}$ & $\begin{array}{r}572 \\
1008 \\
768 \\
1008 \\
720\end{array}$ & $\begin{array}{r}2 \\
12 \\
4 \\
8 \\
12\end{array}$ & $\begin{array}{l}15 \\
20 \\
16 \\
16 \\
18\end{array}$ & $\begin{array}{l}47 \\
79 \\
63 \\
81 \\
55\end{array}$ & $\begin{array}{l}19 \\
35 \\
28 \\
37 \\
19\end{array}$ & $\begin{array}{l}656 \\
657 \\
658 \\
659 \\
660\end{array}$ & $\begin{array}{l}0 \\
0 \\
0 \\
0 \\
0\end{array}$ & $\begin{array}{l}0 \\
0 \\
0 \\
0 \\
0\end{array}$ & $\begin{array}{r}1008 \\
888 \\
1152 \\
660 \\
1728\end{array}$ & $\begin{array}{r}12 \\
8 \\
8 \\
2 \\
24\end{array}$ & $\begin{array}{r}32 \\
16 \\
8 \\
33 \\
16\end{array}$ & $\begin{array}{r}79 \\
71 \\
93 \\
55 \\
133\end{array}$ & $\begin{array}{l}32 \\
32 \\
45 \\
17 \\
63\end{array}$ \\
\hline $\begin{array}{l}576 \\
577 \\
578 \\
579 \\
580\end{array}$ & $\begin{array}{l}0 \\
2 \\
0 \\
2 \\
0\end{array}$ & $\begin{array}{l}0 \\
2 \\
2 \\
0 \\
0\end{array}$ & $\begin{array}{r}1152 \\
578 \\
918 \\
776 \\
1080\end{array}$ & $\begin{array}{r}48 \\
2 \\
36 \\
4 \\
12\end{array}$ & $\begin{array}{r}16 \\
8 \\
16 \\
24 \\
16\end{array}$ & $\begin{array}{l}73 \\
47 \\
59 \\
63 \\
85\end{array}$ & $\begin{array}{l}23 \\
22 \\
26 \\
24 \\
: 9\end{array}$ & $\begin{array}{l}661 \\
662 \\
663 \\
664 \\
665\end{array}$ & $\begin{array}{l}2 \\
0 \\
0 \\
0 \\
0\end{array}$ & $\begin{array}{l}2 \\
0 \\
0 \\
0 \\
0\end{array}$ & $\begin{array}{r}662 \\
996 \\
1008 \\
1008 \\
960\end{array}$ & $\begin{array}{l}2 \\
4 \\
8 \\
8 \\
8\end{array}$ & $\begin{array}{l}18 \\
22 \\
16 \\
20 \\
24\end{array}$ & $\begin{array}{l}54 \\
82 \\
81 \\
81 \\
77\end{array}$ & $\begin{array}{l}23 \\
36 \\
33 \\
36 \\
33\end{array}$ \\
\hline $\begin{array}{l}581 \\
582 \\
583 \\
584 \\
585\end{array}$ & $\begin{array}{l}0 \\
0 \\
0 \\
0 \\
0\end{array}$ & $\begin{array}{l}0 \\
0 \\
0 \\
0 \\
0\end{array}$ & $\begin{array}{r}672 \\
1176 \\
648 \\
888 \\
1008\end{array}$ & $\begin{array}{r}4 \\
8 \\
4 \\
8 \\
16\end{array}$ & $\begin{array}{r}28 \\
16 \\
8 \\
32 \\
16\end{array}$ & $\begin{array}{l}55 \\
95 \\
53 \\
71 \\
77\end{array}$ & $\begin{array}{l}21 \\
44 \\
23 \\
28 \\
35\end{array}$ & $\begin{array}{l}666 \\
667 \\
668 \\
669 \\
670\end{array}$ & $\begin{array}{l}0 \\
0 \\
0 \\
2 \\
0\end{array}$ & $\begin{array}{l}0 \\
0 \\
0 \\
0 \\
0\end{array}$ & $\begin{array}{r}1368 \\
720 \\
1008 \\
896 \\
1224\end{array}$ & $\begin{array}{r}16 \\
4 \\
6 \\
4 \\
8\end{array}$ & $\begin{array}{l}20 \\
12 \\
22 \\
12 \\
12\end{array}$ & $\begin{array}{r}107 \\
59 \\
82 \\
73 \\
99\end{array}$ & $\begin{array}{l}49 \\
26 \\
36 \\
34 \\
47\end{array}$ \\
\hline $\begin{array}{l}586 \\
587 \\
588 \\
589 \\
590\end{array}$ & $\begin{array}{l}0 \\
0 \\
0 \\
4 \\
0\end{array}$ & $\begin{array}{l}2 \\
0 \\
0 \\
0 \\
0\end{array}$ & $\begin{array}{r}882 \\
588 \\
1344 \\
640 \\
1080\end{array}$ & $\begin{array}{r}4 \\
2 \\
48 \\
4 \\
8\end{array}$ & $\begin{array}{l}18 \\
21 \\
12 \\
16 \\
20\end{array}$ & $\begin{array}{l}72 \\
49 \\
89 \\
51 \\
87\end{array}$ & $\begin{array}{l}32 \\
18 \\
42 \\
22 \\
39\end{array}$ & $\begin{array}{l}671 \\
672 \\
673 \\
674 \\
675\end{array}$ & $\begin{array}{l}0 \\
0 \\
2 \\
0 \\
0\end{array}$ & $\begin{array}{l}0 \\
0 \\
2 \\
2 \\
0\end{array}$ & $\begin{array}{r}744 \\
1536 \\
674 \\
1014 \\
1080\end{array}$ & $\begin{array}{r}4 \\
32 \\
2 \\
4 \\
36\end{array}$ & $\begin{array}{l}30 \\
16 \\
12 \\
24 \\
18\end{array}$ & $\begin{array}{r}61 \\
113 \\
55 \\
83 \\
73\end{array}$ & $\begin{array}{l}16 \\
53 \\
25 \\
36 \\
31\end{array}$ \\
\hline $\begin{array}{l}591 \\
592 \\
593 \\
594 \\
595\end{array}$ & $\begin{array}{l}0 \\
0 \\
0 \\
0 \\
0\end{array}$ & $\begin{array}{l}0 \\
0 \\
2 \\
0 \\
0\end{array}$ & $\begin{array}{r}792 \\
912 \\
594 \\
1296 \\
864\end{array}$ & $\begin{array}{r}4 \\
12 \\
2 \\
24 \\
8\end{array}$ & $\begin{array}{r}22 \\
8 \\
24 \\
24 \\
12\end{array}$ & $\begin{array}{l}65 \\
71 \\
49 \\
97 \\
69\end{array}$ & $\begin{array}{l}22 \\
34 \\
19 \\
4.3 \\
31\end{array}$ & $\begin{array}{l}676 \\
677 \\
678 \\
679 \\
680\end{array}$ & $\begin{array}{l}0 \\
0 \\
0 \\
4 \\
0\end{array}$ & $\begin{array}{l}0 \\
2 \\
0 \\
0 \\
0\end{array}$ & $\begin{array}{r}1092 \\
678 \\
1368 \\
784 \\
1296\end{array}$ & $\begin{array}{r}42 \\
2 \\
8 \\
4 \\
16\end{array}$ & $\begin{array}{l}12 \\
30 \\
20 \\
18 \\
24\end{array}$ & $\begin{array}{r}71 \\
56 \\
111 \\
63 \\
101\end{array}$ & $\begin{array}{l}33 \\
21 \\
51 \\
23 \\
45\end{array}$ \\
\hline $\begin{array}{l}596 \\
597 \\
598 \\
599 \\
600\end{array}$ & $\begin{array}{l}0 \\
2 \\
0 \\
0 \\
0\end{array}$ & $\begin{array}{l}0 \\
0 \\
0 \\
0 \\
0\end{array}$ & $\begin{array}{r}900 \\
800 \\
1008 \\
600 \\
1440\end{array}$ & $\begin{array}{r}6 \\
4 \\
8 \\
2 \\
48\end{array}$ & $\begin{array}{r}28 \\
12 \\
8 \\
25 \\
16\end{array}$ & $\begin{array}{l}73 \\
65 \\
81 \\
50 \\
97\end{array}$ & $\begin{array}{l}30 \\
30 \\
39 \\
13 \\
45\end{array}$ & $\begin{array}{l}681 \\
682 \\
683 \\
684 \\
685\end{array}$ & $\begin{array}{l}0 \\
0 \\
0 \\
0 \\
0\end{array}$ & $\begin{array}{l}0 \\
0 \\
0 \\
0 \\
4\end{array}$ & $\begin{array}{r}912 \\
1152 \\
684 \\
1440 \\
828\end{array}$ & $\begin{array}{r}4 \\
8 \\
2 \\
24 \\
4\end{array}$ & $\begin{array}{l}20 \\
12 \\
15 \\
24 \\
12\end{array}$ & $\begin{array}{r}75 \\
93 \\
57 \\
109 \\
67\end{array}$ & $\begin{array}{l}33 \\
44 \\
24 \\
49 \\
31\end{array}$ \\
\hline $\begin{array}{l}601 \\
602 \\
603 \\
604 \\
605\end{array}$ & $\begin{array}{l}2 \\
0 \\
0 \\
0 \\
0\end{array}$ & $\begin{array}{l}2 \\
0 \\
0 \\
0 \\
0\end{array}$ & $\begin{array}{r}602 \\
1056 \\
816 \\
912 \\
792\end{array}$ & $\begin{array}{r}2 \\
8 \\
8 \\
6 \\
24\end{array}$ & $\begin{array}{l}20 \\
24 \\
12 \\
14 \\
24\end{array}$ & $\begin{array}{l}49 \\
85 \\
65 \\
74 \\
55\end{array}$ & $\begin{array}{l}20 \\
37 \\
29 \\
34 \\
22\end{array}$ & $\begin{array}{l}686 \\
687 \\
688 \\
689 \\
690\end{array}$ & $\begin{array}{l}0 \\
2 \\
0 \\
0 \\
0\end{array}$ & $\begin{array}{l}0 \\
0 \\
0 \\
4 \\
0\end{array}$ & $\begin{array}{r}1176 \\
920 \\
1056 \\
756 \\
1728\end{array}$ & $\begin{array}{r}28 \\
4 \\
12 \\
4 \\
16\end{array}$ & $\begin{array}{l}28 \\
12 \\
12 \\
40 \\
16\end{array}$ & $\begin{array}{r}85 \\
75 \\
83 \\
61 \\
137\end{array}$ & $\begin{array}{l}36 \\
32 \\
39 \\
21 \\
65\end{array}$ \\
\hline $\begin{array}{l}606 \\
607 \\
608 \\
609 \\
610\end{array}$ & $\begin{array}{l}0 \\
2 \\
0 \\
0 \\
0\end{array}$ & $\begin{array}{l}0 \\
0 \\
0 \\
0 \\
4\end{array}$ & $\begin{array}{r}1224 \\
608 \\
960 \\
960 \\
1116\end{array}$ & $\begin{array}{r}8 \\
2 \\
16 \\
8 \\
8\end{array}$ & $\begin{array}{l}12 \\
13 \\
24 \\
16 \\
12\end{array}$ & $\begin{array}{l}99 \\
50 \\
73 \\
77 \\
89\end{array}$ & $\begin{array}{l}47 \\
19 \\
31 \\
35 \\
42\end{array}$ & $\begin{array}{l}691 \\
692 \\
693 \\
694 \\
695\end{array}$ & $\begin{array}{l}2 \\
0 \\
0 \\
0 \\
0\end{array}$ & $\begin{array}{l}0 \\
0 \\
0 \\
0 \\
0\end{array}$ & $\begin{array}{r}692 \\
1044 \\
1152 \\
1044 \\
840\end{array}$ & $\begin{array}{r}2 \\
6 \\
16 \\
4 \\
4\end{array}$ & $\begin{array}{l}15 \\
28 \\
16 \\
10 \\
24\end{array}$ & $\begin{array}{l}57 \\
85 \\
89 \\
86 \\
69\end{array}$ & $\begin{array}{l}24 \\
36 \\
41 \\
41 \\
23\end{array}$ \\
\hline $\begin{array}{l}611 \\
612 \\
613 \\
614 \\
615\end{array}$ & $\begin{array}{l}0 \\
0 \\
2 \\
0 \\
0\end{array}$ & $\begin{array}{l}0 \\
0 \\
2 \\
0 \\
0\end{array}$ & $\begin{array}{r}672 \\
1296 \\
614 \\
924 \\
1008\end{array}$ & $\begin{array}{r}4 \\
24 \\
2 \\
4 \\
8\end{array}$ & $\begin{array}{l}30 \\
16 \\
10 \\
34 \\
20\end{array}$ & $\begin{array}{l}55 \\
97 \\
50 \\
76 \\
81\end{array}$ & $\begin{array}{l}18 \\
45 \\
23 \\
30 \\
31\end{array}$ & $\begin{array}{l}696 \\
697 \\
698 \\
699 \\
700\end{array}$ & $\begin{array}{l}0 \\
0 \\
0 \\
0 \\
0\end{array}$ & $\begin{array}{l}0 \\
4 \\
2 \\
0 \\
0\end{array}$ & $\begin{array}{r}1440 \\
756 \\
1050 \\
936 \\
1440\end{array}$ & $\begin{array}{r}16 \\
4 \\
4 \\
4 \\
36\end{array}$ & $\begin{array}{r}24 \\
8 \\
26 \\
30 \\
12\end{array}$ & $\begin{array}{r}113 \\
61 \\
86 \\
77 \\
103\end{array}$ & $\begin{array}{l}51 \\
29 \\
37 \\
29 \\
49\end{array}$ \\
\hline $\begin{array}{l}616 \\
617 \\
618 \\
619 \\
620\end{array}$ & $\begin{array}{l}0 \\
0 \\
0 \\
2 \\
0\end{array}$ & $\begin{array}{l}0 \\
2 \\
0 \\
0 \\
0\end{array}$ & $\begin{array}{r}1152 \\
618 \\
1248 \\
620 \\
1152\end{array}$ & $\begin{array}{r}16 \\
2 \\
8 \\
2 \\
12\end{array}$ & $\begin{array}{l}16 \\
12 \\
12 \\
15 \\
24\end{array}$ & $\begin{array}{r}89 \\
51 \\
101 \\
51 \\
91\end{array}$ & $\begin{array}{l}41 \\
23 \\
48 \\
21 \\
40\end{array}$ & $\begin{array}{l}701 \\
702 \\
703 \\
704 \\
705\end{array}$ & $\begin{array}{l}0 \\
0 \\
4 \\
0 \\
0\end{array}$ & $\begin{array}{l}2 \\
0 \\
0 \\
0 \\
0\end{array}$ & $\begin{array}{r}702 \\
1512 \\
760 \\
1152 \\
1152\end{array}$ & $\begin{array}{r}2 \\
24 \\
4 \\
24 \\
8\end{array}$ & $\begin{array}{l}34 \\
12 \\
14 \\
24 \\
24\end{array}$ & $\begin{array}{r}58 \\
115 \\
61 \\
85 \\
93\end{array}$ & $\begin{array}{l}21 \\
55 \\
24 \\
37 \\
41\end{array}$ \\
\hline $\begin{array}{l}621 \\
622 \\
623 \\
624 \\
625\end{array}$ & $\begin{array}{l}0 \\
0 \\
0 \\
0 \\
0\end{array}$ & $\begin{array}{l}0 \\
0 \\
0 \\
0 \\
2\end{array}$ & $\begin{array}{r}864 \\
936 \\
720 \\
1344 \\
750\end{array}$ & $\begin{array}{r}12 \\
4 \\
4 \\
24 \\
30\end{array}$ & $\begin{array}{l}24 \\
12 \\
22 \\
16 \\
10\end{array}$ & $\begin{array}{r}67 \\
77 \\
59 \\
101 \\
48\end{array}$ & $\begin{array}{l}28 \\
36 \\
19 \\
47 \\
22\end{array}$ & $\begin{array}{l}706 \\
707 \\
708 \\
709 \\
710\end{array}$ & $\begin{array}{l}0 \\
0 \\
0 \\
2 \\
0\end{array}$ & $\begin{array}{l}2 \\
0 \\
0 \\
2 \\
0\end{array}$ & $\begin{array}{r}1062 \\
816 \\
1440 \\
710 \\
1296\end{array}$ & $\begin{array}{r}4 \\
4 \\
12 \\
2 \\
8\end{array}$ & $\begin{array}{r}24 \\
18 \\
8 \\
10 \\
32\end{array}$ & $\begin{array}{r}87 \\
67 \\
115 \\
58 \\
105\end{array}$ & $\begin{array}{l}38 \\
28 \\
56 \\
27 \\
45\end{array}$ \\
\hline $\begin{array}{l}626 \\
627 \\
628 \\
629 \\
630\end{array}$ & $\begin{array}{l}0 \\
0 \\
0 \\
0 \\
0\end{array}$ & $\begin{array}{l}2 \\
0 \\
0 \\
4 \\
0\end{array}$ & $\begin{array}{r}942 \\
960 \\
948 \\
684 \\
1728\end{array}$ & $\begin{array}{r}4 \\
8 \\
6 \\
4 \\
32\end{array}$ & $\begin{array}{l}36 \\
12 \\
12 \\
36 \\
16\end{array}$ & $\begin{array}{r}77 \\
77 \\
77 \\
55 \\
129\end{array}$ & $\begin{array}{l}30 \\
35 \\
36 \\
19 \\
61\end{array}$ & $\begin{array}{l}711 \\
712 \\
713 \\
714 \\
715\end{array}$ & $\begin{array}{l}0 \\
0 \\
0 \\
0 \\
0\end{array}$ & $\begin{array}{l}0 \\
0 \\
0 \\
0 \\
0\end{array}$ & $\begin{array}{r}960 \\
1080 \\
768 \\
1728 \\
1008\end{array}$ & $\begin{array}{r}8 \\
8 \\
4 \\
16 \\
8\end{array}$ & $\begin{array}{l}20 \\
16 \\
24 \\
24 \\
12\end{array}$ & $\begin{array}{r}77 \\
87 \\
63 \\
137 \\
81\end{array}$ & $\begin{array}{l}29 \\
40 \\
26 \\
63 \\
37\end{array}$ \\
\hline $\begin{array}{l}631 \\
632 \\
633 \\
634 \\
635\end{array}$ & $\begin{array}{l}2 \\
0 \\
2 \\
0 \\
0\end{array}$ & $\begin{array}{l}0 \\
0 \\
0 \\
2 \\
0\end{array}$ & $\begin{array}{l}632 \\
960 \\
848 \\
954 \\
768\end{array}$ & $\begin{array}{l}2 \\
8 \\
4 \\
4 \\
4\end{array}$ & $\begin{array}{l}13 \\
16 \\
20 \\
14 \\
30\end{array}$ & $\begin{array}{l}52 \\
77 \\
69 \\
78 \\
63\end{array}$ & $\begin{array}{l}20 \\
35 \\
30 \\
36 \\
22\end{array}$ & $\begin{array}{l}716 \\
717 \\
718 \\
719 \\
720\end{array}$ & $\begin{array}{l}0 \\
0 \\
0 \\
0 \\
0\end{array}$ & $\begin{array}{l}0 \\
0 \\
0 \\
0 \\
0\end{array}$ & $\begin{array}{r}1080 \\
960 \\
1080 \\
720 \\
1728\end{array}$ & $\begin{array}{r}6 \\
4 \\
4 \\
2 \\
48\end{array}$ & $\begin{array}{l}30 \\
16 \\
12 \\
31 \\
16\end{array}$ & $\begin{array}{r}88 \\
79 \\
89 \\
60 \\
121\end{array}$ & $\begin{array}{l}37 \\
36 \\
42 \\
15 \\
57\end{array}$ \\
\hline $\begin{array}{l}636 \\
637 \\
638 \\
639 \\
640\end{array}$ & $\begin{array}{l}0 \\
4 \\
0 \\
0 \\
0\end{array}$ & $\begin{array}{l}0 \\
0 \\
0 \\
0 \\
0\end{array}$ & $\begin{array}{r}1296 \\
784 \\
1080 \\
864 \\
1152\end{array}$ & $\begin{array}{r}12 \\
16 \\
8 \\
8 \\
32\end{array}$ & $\begin{array}{l}20 \\
12 \\
20 \\
14 \\
16\end{array}$ & $\begin{array}{r}103 \\
57 \\
87 \\
69 \\
81\end{array}$ & $\begin{array}{l}47 \\
26 \\
39 \\
28 \\
37\end{array}$ & $\begin{array}{l}721 \\
722 \\
723 \\
724 \\
725\end{array}$ & $\begin{array}{l}4 \\
0 \\
2 \\
0 \\
0\end{array}$ & $\begin{array}{l}0 \\
0 \\
0 \\
0 \\
4\end{array}$ & $\begin{array}{r}832 \\
1140 \\
968 \\
1092 \\
900\end{array}$ & $\begin{array}{r}4 \\
40 \\
4 \\
6 \\
12\end{array}$ & $\begin{array}{l}16 \\
18 \\
12 \\
20 \\
24\end{array}$ & $\begin{array}{l}67 \\
76 \\
79 \\
89 \\
69\end{array}$ & $\begin{array}{l}30 \\
34 \\
36 \\
40 \\
29\end{array}$ \\
\hline
\end{tabular}




\begin{tabular}{|c|c|c|c|c|c|c|c|c|c|c|c|c|c|c|c|}
\hline$n$ & $\nu_{1}(n)$ & $\nu_{2}(n)$ & $\mu(n)$ & $\sigma_{0}(n)$ & $h(-4 n)$ & $g_{0}(n)$ & $g^{*}(n)$ & $n$ & $\nu_{1}(n)$ & $\nu_{2}(n)$ & $\mu(n)$ & $\sigma_{0}(n)$ & $h(-4 n)$ & $g_{0}(n)$ & $g^{*}(n)$ \\
\hline $\begin{array}{l}726 \\
727 \\
728 \\
729 \\
730\end{array}$ & $\begin{array}{l}0 \\
2 \\
0 \\
0 \\
0\end{array}$ & $\begin{array}{l}0 \\
0 \\
0 \\
0 \\
4\end{array}$ & $\begin{array}{r}1584 \\
728 \\
1344 \\
972 \\
1332\end{array}$ & $\begin{array}{r}48 \\
2 \\
16 \\
36 \\
8\end{array}$ & $\begin{array}{l}20 \\
13 \\
24 \\
18 \\
12\end{array}$ & $\begin{array}{r}109 \\
60 \\
105 \\
64 \\
107\end{array}$ & $\begin{array}{l}50 \\
24 \\
47 \\
28 \\
51\end{array}$ & $\begin{array}{l}811 \\
812 \\
813 \\
814 \\
815\end{array}$ & $\begin{array}{l}2 \\
0 \\
2 \\
0 \\
0\end{array}$ & $\begin{array}{l}0 \\
0 \\
0 \\
0 \\
0\end{array}$ & $\begin{array}{r}812 \\
1440 \\
1088 \\
1368 \\
984\end{array}$ & $\begin{array}{r}2 \\
12 \\
4 \\
8 \\
4\end{array}$ & $\begin{array}{l}21 \\
24 \\
12 \\
12 \\
30\end{array}$ & $\begin{array}{r}67 \\
115 \\
89 \\
111 \\
81\end{array}$ & $\begin{array}{l}27 \\
52 \\
42 \\
53 \\
26\end{array}$ \\
\hline $\begin{array}{l}731 \\
732 \\
733 \\
734 \\
735\end{array}$ & $\begin{array}{l}0 \\
0 \\
2 \\
0 \\
0\end{array}$ & $\begin{array}{l}0 \\
0 \\
2 \\
0 \\
0\end{array}$ & $\begin{array}{r}792 \\
1488 \\
734 \\
1104 \\
1344\end{array}$ & $\begin{array}{r}4 \\
12 \\
2 \\
4 \\
32\end{array}$ & $\begin{array}{l}36 \\
16 \\
14 \\
40 \\
16\end{array}$ & $\begin{array}{r}65 \\
119 \\
60 \\
91 \\
97\end{array}$ & $\begin{array}{l}21 \\
56 \\
27 \\
36 \\
41\end{array}$ & $\begin{array}{l}816 \\
817 \\
818 \\
819 \\
820\end{array}$ & $\begin{array}{l}0 \\
4 \\
0 \\
0 \\
0\end{array}$ & $\begin{array}{l}0 \\
0 \\
2 \\
0 \\
0\end{array}$ & $\begin{array}{r}1728 \\
880 \\
1230 \\
1344 \\
1512\end{array}$ & $\begin{array}{r}24 \\
4 \\
4 \\
16 \\
12\end{array}$ & $\begin{array}{l}24 \\
12 \\
28 \\
24 \\
16\end{array}$ & $\begin{array}{r}133 \\
71 \\
101 \\
105 \\
121\end{array}$ & $\begin{array}{l}61 \\
33 \\
44 \\
45 \\
57\end{array}$ \\
\hline $\begin{array}{l}736 \\
737 \\
738 \\
739 \\
740\end{array}$ & $\begin{array}{l}0 \\
0 \\
0 \\
2 \\
0\end{array}$ & $\begin{array}{l}0 \\
0 \\
0 \\
0 \\
0\end{array}$ & $\begin{array}{r}1152 \\
816 \\
1512 \\
740 \\
1368\end{array}$ & $\begin{array}{r}16 \\
4 \\
16 \\
2 \\
12\end{array}$ & $\begin{array}{l}16 \\
20 \\
16 \\
15 \\
32\end{array}$ & $\begin{array}{r}89 \\
67 \\
119 \\
61 \\
109\end{array}$ & $\begin{array}{l}41 \\
29 \\
56 \\
26 \\
47\end{array}$ & $\begin{array}{l}821 \\
822 \\
823 \\
824 \\
825\end{array}$ & $\begin{array}{l}0 \\
0 \\
2 \\
0 \\
0\end{array}$ & $\begin{array}{l}2 \\
0 \\
0 \\
0 \\
0\end{array}$ & $\begin{array}{r}822 \\
1656 \\
824 \\
1248 \\
1440\end{array}$ & $\begin{array}{r}2 \\
8 \\
2 \\
8 \\
24\end{array}$ & $\begin{array}{r}30 \\
20 \\
9 \\
40 \\
24\end{array}$ & $\begin{array}{r}68 \\
135 \\
68 \\
101 \\
109\end{array}$ & $\begin{array}{l}27 \\
63 \\
30 \\
41 \\
49\end{array}$ \\
\hline $\begin{array}{l}741 \\
742 \\
743 \\
744 \\
745\end{array}$ & $\begin{array}{l}4 \\
0 \\
0 \\
0 \\
0\end{array}$ & $\begin{array}{l}0 \\
0 \\
0 \\
0 \\
4\end{array}$ & $\begin{array}{r}1120 \\
1296 \\
744 \\
1536 \\
900\end{array}$ & $\begin{array}{r}8 \\
8 \\
2 \\
16 \\
4\end{array}$ & $\begin{array}{r}24 \\
8 \\
21 \\
24 \\
16\end{array}$ & $\begin{array}{r}89 \\
105 \\
62 \\
121 \\
73\end{array}$ & $\begin{array}{l}39 \\
51 \\
21 \\
55 \\
33\end{array}$ & $\begin{array}{l}826 \\
827 \\
828 \\
829 \\
830\end{array}$ & $\begin{array}{l}0 \\
0 \\
0 \\
2 \\
0\end{array}$ & $\begin{array}{l}0 \\
0 \\
0 \\
2 \\
0\end{array}$ & $\begin{array}{r}1440 \\
828 \\
1728 \\
830 \\
1512\end{array}$ & $\begin{array}{r}8 \\
2 \\
24 \\
2 \\
8\end{array}$ & $\begin{array}{l}12 \\
21 \\
12 \\
22 \\
20\end{array}$ & $\begin{array}{r}117 \\
69 \\
133 \\
68 \\
123\end{array}$ & $\begin{array}{l}56 \\
28 \\
64 \\
29 \\
57\end{array}$ \\
\hline $\begin{array}{l}746 \\
747 \\
748 \\
749 \\
750\end{array}$ & $\begin{array}{l}0 \\
0 \\
0 \\
0 \\
0\end{array}$ & $\begin{array}{l}2 \\
0 \\
0 \\
0 \\
0\end{array}$ & $\begin{array}{r}1122 \\
1008 \\
1296 \\
864 \\
1800\end{array}$ & $\begin{array}{r}4 \\
8 \\
12 \\
4 \\
40\end{array}$ & $\begin{array}{l}26 \\
18 \\
12 \\
32 \\
20\end{array}$ & $\begin{array}{r}92 \\
81 \\
103 \\
71 \\
131\end{array}$ & $\begin{array}{l}40 \\
35 \\
49 \\
28 \\
61\end{array}$ & $\begin{array}{l}831 \\
832 \\
833 \\
834 \\
835\end{array}$ & $\begin{array}{l}2 \\
0 \\
0 \\
0 \\
0\end{array}$ & $\begin{array}{l}0 \\
0 \\
0 \\
0 \\
0\end{array}$ & $\begin{array}{l}1112 \\
1344 \\
1008 \\
1680 \\
1008\end{array}$ & $\begin{array}{r}4 \\
24 \\
16 \\
8 \\
4\end{array}$ & $\begin{array}{l}28 \\
16 \\
24 \\
16 \\
18\end{array}$ & $\begin{array}{r}91 \\
101 \\
77 \\
137 \\
83\end{array}$ & $\begin{array}{l}32 \\
47 \\
33 \\
65 \\
36\end{array}$ \\
\hline $\begin{array}{l}751 \\
752 \\
753 \\
754 \\
755\end{array}$ & $\begin{array}{l}2 \\
0 \\
0 \\
0 \\
0\end{array}$ & $\begin{array}{l}0 \\
0 \\
0 \\
4 \\
0\end{array}$ & $\begin{array}{r}752 \\
1152 \\
1008 \\
1260 \\
912\end{array}$ & $\begin{array}{r}2 \\
12 \\
4 \\
8 \\
4\end{array}$ & $\begin{array}{l}15 \\
20 \\
12 \\
20 \\
36\end{array}$ & $\begin{array}{r}62 \\
91 \\
83 \\
101 \\
75\end{array}$ & $\begin{array}{l}24 \\
41 \\
39 \\
46 \\
26\end{array}$ & $\begin{array}{l}836 \\
837 \\
838 \\
839 \\
840\end{array}$ & $\begin{array}{l}0 \\
0 \\
0 \\
0 \\
0\end{array}$ & $\begin{array}{l}0 \\
0 \\
0 \\
0 \\
0\end{array}$ & $\begin{array}{r}1440 \\
1152 \\
1260 \\
840 \\
2304\end{array}$ & $\begin{array}{r}12 \\
12 \\
4 \\
2 \\
32\end{array}$ & $\begin{array}{l}40 \\
12 \\
14 \\
33 \\
16\end{array}$ & $\begin{array}{r}115 \\
91 \\
104 \\
70 \\
177\end{array}$ & $\begin{array}{l}48 \\
43 \\
49 \\
19 \\
85\end{array}$ \\
\hline $\begin{array}{l}756 \\
757 \\
758 \\
759 \\
760\end{array}$ & $\begin{array}{l}0 \\
2 \\
0 \\
0 \\
0\end{array}$ & $\begin{array}{l}0 \\
2 \\
0 \\
0 \\
0\end{array}$ & $\begin{array}{r}1728 \\
758 \\
1140 \\
1152 \\
1440\end{array}$ & $\begin{array}{r}36 \\
2 \\
4 \\
8 \\
16\end{array}$ & $\begin{array}{r}24 \\
10 \\
22 \\
24 \\
8\end{array}$ & $\begin{array}{r}127 \\
62 \\
94 \\
93 \\
113\end{array}$ & $\begin{array}{l}58 \\
29 \\
42 \\
35 \\
55\end{array}$ & $\begin{array}{l}841 \\
842 \\
843 \\
844 \\
845\end{array}$ & $\begin{array}{l}0 \\
0 \\
0 \\
0 \\
0\end{array}$ & $\begin{array}{l}2 \\
2 \\
0 \\
0 \\
4\end{array}$ & $\begin{array}{r}870 \\
1266 \\
1128 \\
1272 \\
1092\end{array}$ & $\begin{array}{r}30 \\
4 \\
4 \\
6 \\
28\end{array}$ & $\begin{array}{l}14 \\
26 \\
18 \\
18 \\
28\end{array}$ & $\begin{array}{r}58 \\
104 \\
93 \\
104 \\
77\end{array}$ & $\begin{array}{l}26 \\
46 \\
41 \\
48 \\
32\end{array}$ \\
\hline $\begin{array}{l}761 \\
762 \\
763 \\
764 \\
765\end{array}$ & $\begin{array}{l}0 \\
0 \\
4 \\
0 \\
0\end{array}$ & $\begin{array}{l}2 \\
0 \\
0 \\
0 \\
0\end{array}$ & $\begin{array}{r}762 \\
1536 \\
880 \\
1152 \\
1296\end{array}$ & $\begin{array}{r}2 \\
8 \\
4 \\
6 \\
16\end{array}$ & $\begin{array}{l}40 \\
12 \\
12 \\
26 \\
16\end{array}$ & $\begin{array}{r}63 \\
125 \\
71 \\
94 \\
101\end{array}$ & $\begin{array}{l}22 \\
60 \\
32 \\
41 \\
47\end{array}$ & $\begin{array}{l}846 \\
847 \\
848 \\
849 \\
850\end{array}$ & $\begin{array}{l}0 \\
0 \\
0 \\
2 \\
0\end{array}$ & $\begin{array}{l}0 \\
0 \\
0 \\
0 \\
4\end{array}$ & $\begin{array}{l}1728 \\
1056 \\
1296 \\
1136 \\
1620\end{array}$ & $\begin{array}{r}16 \\
24 \\
12 \\
4 \\
24\end{array}$ & $\begin{array}{l}32 \\
10 \\
24 \\
28 \\
16\end{array}$ & $\begin{array}{r}137 \\
77 \\
103 \\
93 \\
123\end{array}$ & $\begin{array}{l}61 \\
34 \\
46 \\
40 \\
58\end{array}$ \\
\hline $\begin{array}{l}766 \\
767 \\
768 \\
769 \\
770\end{array}$ & $\begin{array}{l}0 \\
0 \\
0 \\
2 \\
0\end{array}$ & $\begin{array}{l}0 \\
0 \\
0 \\
2 \\
0\end{array}$ & $\begin{array}{r}1152 \\
840 \\
1536 \\
770 \\
1728\end{array}$ & $\begin{array}{r}4 \\
4 \\
48 \\
2 \\
16\end{array}$ & $\begin{array}{l}24 \\
22 \\
16 \\
20 \\
32\end{array}$ & $\begin{array}{r}95 \\
69 \\
105 \\
63 \\
137\end{array}$ & $\begin{array}{l}42 \\
24 \\
49 \\
27 \\
61\end{array}$ & $\begin{array}{l}851 \\
852 \\
853 \\
854 \\
855\end{array}$ & $\begin{array}{l}0 \\
0 \\
2 \\
0 \\
0\end{array}$ & $\begin{array}{l}0 \\
0 \\
2 \\
0 \\
0\end{array}$ & $\begin{array}{r}912 \\
1728 \\
854 \\
1488 \\
1440\end{array}$ & $\begin{array}{r}4 \\
12 \\
2 \\
8 \\
16\end{array}$ & $\begin{array}{l}30 \\
16 \\
10 \\
44 \\
16\end{array}$ & $\begin{array}{r}75 \\
139 \\
70 \\
121 \\
113\end{array}$ & $\begin{array}{l}28 \\
66 \\
33 \\
50 \\
49\end{array}$ \\
\hline $\begin{array}{l}771 \\
772 \\
773 \\
774 \\
775\end{array}$ & $\begin{array}{l}0 \\
0 \\
0 \\
0 \\
0\end{array}$ & $\begin{array}{l}0 \\
0 \\
2 \\
0 \\
0\end{array}$ & $\begin{array}{r}1032 \\
1164 \\
774 \\
1584 \\
960\end{array}$ & $\begin{array}{r}4 \\
6 \\
2 \\
16 \\
12\end{array}$ & $\begin{array}{r}18 \\
8 \\
26 \\
20 \\
12\end{array}$ & $\begin{array}{r}85 \\
95 \\
64 \\
125 \\
75\end{array}$ & $\begin{array}{l}37 \\
46 \\
26 \\
58 \\
32\end{array}$ & $\begin{array}{l}856 \\
857 \\
858 \\
859 \\
860\end{array}$ & $\begin{array}{l}0 \\
0 \\
0 \\
2 \\
0\end{array}$ & $\begin{array}{l}0 \\
2 \\
0 \\
0 \\
0\end{array}$ & $\begin{array}{r}1296 \\
858 \\
2016 \\
860 \\
1584\end{array}$ & $\begin{array}{r}8 \\
2 \\
16 \\
2 \\
12\end{array}$ & $\begin{array}{l}12 \\
32 \\
16 \\
21 \\
28\end{array}$ & $\begin{array}{r}105 \\
71 \\
161 \\
71 \\
127\end{array}$ & $\begin{array}{l}50 \\
28 \\
77 \\
29 \\
57\end{array}$ \\
\hline $\begin{array}{l}776 \\
777 \\
778 \\
779 \\
780\end{array}$ & $\begin{array}{l}0 \\
4 \\
0 \\
0 \\
0\end{array}$ & $\begin{array}{l}0 \\
0 \\
2 \\
0 \\
0\end{array}$ & $\begin{array}{r}1176 \\
1216 \\
1170 \\
840 \\
2016\end{array}$ & $\begin{array}{r}8 \\
8 \\
4 \\
4 \\
24\end{array}$ & $\begin{array}{l}40 \\
16 \\
14 \\
30 \\
24\end{array}$ & $\begin{array}{r}95 \\
97 \\
96 \\
69 \\
157\end{array}$ & $\begin{array}{l}38 \\
45 \\
45 \\
25 \\
73\end{array}$ & $\begin{array}{l}861 \\
862 \\
863 \\
864 \\
865\end{array}$ & $\begin{array}{l}0 \\
0 \\
0 \\
0 \\
0\end{array}$ & $\begin{array}{l}0 \\
0 \\
0 \\
0 \\
4\end{array}$ & $\begin{array}{r}1344 \\
1296 \\
864 \\
1728 \\
1044\end{array}$ & $\begin{array}{r}8 \\
4 \\
2 \\
48 \\
4\end{array}$ & $\begin{array}{r}24 \\
8 \\
21 \\
24 \\
16\end{array}$ & $\begin{array}{r}109 \\
107 \\
72 \\
121 \\
85\end{array}$ & $\begin{array}{l}49 \\
52 \\
26 \\
55 \\
39\end{array}$ \\
\hline $\begin{array}{l}781 \\
782 \\
783 \\
784 \\
785\end{array}$ & $\begin{array}{l}0 \\
0 \\
0 \\
0 \\
0\end{array}$ & $\begin{array}{l}0 \\
0 \\
0 \\
0 \\
4\end{array}$ & $\begin{array}{r}864 \\
1296 \\
1080 \\
1344 \\
948\end{array}$ & $\begin{array}{r}4 \\
8 \\
12 \\
48 \\
4\end{array}$ & $\begin{array}{l}20 \\
24 \\
18 \\
16 \\
16\end{array}$ & $\begin{array}{r}71 \\
105 \\
85 \\
89 \\
77\end{array}$ & $\begin{array}{l}31 \\
47 \\
34 \\
41 \\
35\end{array}$ & $\begin{array}{l}866 \\
867 \\
868 \\
869 \\
870\end{array}$ & $\begin{array}{l}0 \\
0 \\
0 \\
0 \\
0\end{array}$ & $\begin{array}{l}2 \\
0 \\
0 \\
0 \\
0\end{array}$ & $\begin{array}{r}1302 \\
1224 \\
1536 \\
960 \\
2160\end{array}$ & $\begin{array}{r}4 \\
36 \\
12 \\
4 \\
16\end{array}$ & $\begin{array}{l}44 \\
18 \\
16 \\
32 \\
16\end{array}$ & $\begin{array}{r}107 \\
85 \\
123 \\
79 \\
173\end{array}$ & $\begin{array}{l}43 \\
37 \\
58 \\
32 \\
83\end{array}$ \\
\hline $\begin{array}{l}786 \\
787 \\
788 \\
789 \\
790\end{array}$ & $\begin{array}{l}0 \\
2 \\
0 \\
0 \\
0\end{array}$ & $\begin{array}{l}0 \\
0 \\
0 \\
0 \\
0\end{array}$ & $\begin{array}{r}1584 \\
788 \\
1188 \\
1056 \\
1440\end{array}$ & $\begin{array}{l}8 \\
2 \\
6 \\
4 \\
8\end{array}$ & $\begin{array}{l}16 \\
15 \\
20 \\
32 \\
16\end{array}$ & $\begin{array}{r}129 \\
65 \\
97 \\
87 \\
117\end{array}$ & $\begin{array}{l}61 \\
28 \\
44 \\
36 \\
55\end{array}$ & $\begin{array}{l}871 \\
872 \\
873 \\
874 \\
875\end{array}$ & $\begin{array}{l}4 \\
0 \\
0 \\
0 \\
0\end{array}$ & $\begin{array}{l}0 \\
0 \\
0 \\
0 \\
0\end{array}$ & $\begin{array}{r}952 \\
1320 \\
1176 \\
1440 \\
1200\end{array}$ & $\begin{array}{r}4 \\
8 \\
8 \\
8 \\
20\end{array}$ & $\begin{array}{l}22 \\
20 \\
16 \\
20 \\
30\end{array}$ & $\begin{array}{r}77 \\
107 \\
95 \\
117 \\
91\end{array}$ & $\begin{array}{l}28 \\
49 \\
44 \\
54 \\
36\end{array}$ \\
\hline $\begin{array}{l}791 \\
792 \\
793 \\
794 \\
795\end{array}$ & $\begin{array}{l}0 \\
0 \\
4 \\
0 \\
0\end{array}$ & $\begin{array}{l}0 \\
0 \\
4 \\
2 \\
0\end{array}$ & $\begin{array}{r}912 \\
1728 \\
868 \\
1194 \\
1296\end{array}$ & $\begin{array}{r}4 \\
32 \\
4 \\
4 \\
8\end{array}$ & $\begin{array}{r}32 \\
16 \\
8 \\
42 \\
12\end{array}$ & $\begin{array}{r}75 \\
129 \\
69 \\
98 \\
105\end{array}$ & $\begin{array}{l}22 \\
61 \\
33 \\
39 \\
49\end{array}$ & $\begin{array}{l}876 \\
877 \\
878 \\
879 \\
880\end{array}$ & $\begin{array}{l}0 \\
2 \\
0 \\
0 \\
0\end{array}$ & $\begin{array}{l}0 \\
2 \\
0 \\
0 \\
0\end{array}$ & $\begin{array}{r}1776 \\
878 \\
1320 \\
1176 \\
1728\end{array}$ & $\begin{array}{r}12 \\
2 \\
4 \\
4 \\
24\end{array}$ & $\begin{array}{l}24 \\
10 \\
20 \\
22 \\
16\end{array}$ & $\begin{array}{r}143 \\
72 \\
109 \\
97 \\
133\end{array}$ & $\begin{array}{l}66 \\
34 \\
50 \\
38 \\
63\end{array}$ \\
\hline $\begin{array}{l}796 \\
797 \\
798 \\
799 \\
800\end{array}$ & $\begin{array}{l}0 \\
0 \\
0 \\
0 \\
0\end{array}$ & $\begin{array}{l}0 \\
2 \\
0 \\
0 \\
0\end{array}$ & $\begin{array}{r}1200 \\
798 \\
1920 \\
864 \\
1440\end{array}$ & $\begin{array}{r}6 \\
2 \\
16 \\
4 \\
48\end{array}$ & $\begin{array}{l}18 \\
30 \\
16 \\
16 \\
24\end{array}$ & $\begin{array}{r}98 \\
66 \\
153 \\
71 \\
97\end{array}$ & $\begin{array}{l}45 \\
26 \\
73 \\
28 \\
43\end{array}$ & $\begin{array}{l}881 \\
882 \\
883 \\
884 \\
885\end{array}$ & $\begin{array}{l}0 \\
0 \\
2 \\
0 \\
0\end{array}$ & $\begin{array}{l}2 \\
0 \\
0 \\
0 \\
0\end{array}$ & $\begin{array}{r}882 \\
2016 \\
884 \\
1512 \\
1440\end{array}$ & $\begin{array}{r}2 \\
64 \\
2 \\
12 \\
8\end{array}$ & $\begin{array}{r}40 \\
16 \\
9 \\
32 \\
24\end{array}$ & $\begin{array}{r}73 \\
137 \\
73 \\
121 \\
117\end{array}$ & $\begin{array}{l}27 \\
65 \\
34 \\
53 \\
53\end{array}$ \\
\hline $\begin{array}{l}801 \\
802 \\
803 \\
804 \\
805\end{array}$ & $\begin{array}{l}0 \\
0 \\
0 \\
0 \\
0\end{array}$ & $\begin{array}{l}0 \\
2 \\
0 \\
0 \\
0\end{array}$ & $\begin{array}{r}1080 \\
1206 \\
888 \\
1632 \\
1152\end{array}$ & $\begin{array}{r}8 \\
4 \\
4 \\
12 \\
8\end{array}$ & $\begin{array}{l}24 \\
12 \\
30 \\
24 \\
16\end{array}$ & $\begin{array}{r}87 \\
99 \\
73 \\
131 \\
93\end{array}$ & $\begin{array}{l}38 \\
47 \\
27 \\
60 \\
43\end{array}$ & $\begin{array}{l}886 \\
887 \\
888 \\
889 \\
890\end{array}$ & $\begin{array}{l}0 \\
0 \\
0 \\
4 \\
0\end{array}$ & $\begin{array}{l}0 \\
0 \\
0 \\
0 \\
4\end{array}$ & $\begin{array}{r}1332 \\
888 \\
1824 \\
1024 \\
1620\end{array}$ & $\begin{array}{r}4 \\
2 \\
16 \\
4 \\
8\end{array}$ & $\begin{array}{l}18 \\
29 \\
24 \\
16 \\
24\end{array}$ & $\begin{array}{r}110 \\
74 \\
145 \\
83 \\
131\end{array}$ & $\begin{array}{l}51 \\
23 \\
67 \\
38 \\
60\end{array}$ \\
\hline $\begin{array}{l}806 \\
807 \\
808 \\
809 \\
810\end{array}$ & $\begin{array}{l}0 \\
0 \\
0 \\
0 \\
0\end{array}$ & $\begin{array}{l}0 \\
0 \\
0 \\
2 \\
0\end{array}$ & $\begin{array}{r}1344 \\
1080 \\
1224 \\
810 \\
1944\end{array}$ & $\begin{array}{r}8 \\
4 \\
8 \\
2 \\
48\end{array}$ & $\begin{array}{l}28 \\
14 \\
12 \\
32 \\
24\end{array}$ & $\begin{array}{r}109 \\
89 \\
99 \\
67 \\
139\end{array}$ & $\begin{array}{l}48 \\
38 \\
47 \\
26 \\
64\end{array}$ & $\begin{array}{l}891 \\
892 \\
893 \\
894 \\
895\end{array}$ & $\begin{array}{l}0 \\
0 \\
0 \\
0 \\
0\end{array}$ & $\begin{array}{l}0 \\
0 \\
0 \\
0 \\
0\end{array}$ & $\begin{array}{r}1296 \\
1344 \\
960 \\
1800 \\
1080\end{array}$ & $\begin{array}{r}24 \\
6 \\
4 \\
8 \\
4\end{array}$ & $\begin{array}{l}18 \\
14 \\
28 \\
28 \\
16\end{array}$ & $\begin{array}{r}97 \\
110 \\
79 \\
147 \\
89\end{array}$ & $\begin{array}{l}43 \\
52 \\
33 \\
67 \\
37\end{array}$ \\
\hline
\end{tabular}




\begin{tabular}{|c|c|c|c|c|c|c|c|c|c|c|c|c|c|c|c|}
\hline$n$ & $\nu_{1}(n)$ & $\nu_{2}(n)$ & $\mu_{0}(n)$ & $\sigma_{0}(n)$ & $h(-4 n)$ & $g_{0}(n)$ & $g^{*}(n)$ & $n$ & $\nu_{1}(n)$ & $\nu_{2}(n)$ & $\mu_{0}(n)$ & $\sigma_{0}(n)$ & $h(-4 n)$ & $g_{0}(n)$ & $g^{*}(n)$ \\
\hline $\begin{array}{l}896 \\
897 \\
898 \\
899 \\
900\end{array}$ & $\begin{array}{l}0 \\
0 \\
0 \\
0 \\
0\end{array}$ & $\begin{array}{l}0 \\
0 \\
2 \\
0 \\
0\end{array}$ & $\begin{array}{r}1536 \\
1344 \\
1350 \\
960 \\
2160\end{array}$ & $\begin{array}{r}32 \\
8 \\
4 \\
4 \\
72\end{array}$ & $\begin{array}{l}32 \\
16 \\
12 \\
42 \\
16\end{array}$ & $\begin{array}{r}113 \\
109 \\
111 \\
79 \\
145\end{array}$ & $\begin{array}{l}49 \\
51 \\
53 \\
26 \\
69\end{array}$ & $\begin{array}{l}961 \\
962 \\
963 \\
964 \\
965\end{array}$ & $\begin{array}{l}2 \\
0 \\
0 \\
0 \\
0\end{array}$ & $\begin{array}{l}0 \\
4 \\
0 \\
0 \\
4\end{array}$ & $\begin{array}{r}992 \\
1596 \\
1296 \\
1452 \\
1164\end{array}$ & $\begin{array}{r}32 \\
8 \\
8 \\
6 \\
4\end{array}$ & $\begin{array}{l}16 \\
28 \\
18 \\
24 \\
44\end{array}$ & $\begin{array}{r}67 \\
129 \\
105 \\
119 \\
95\end{array}$ & $\begin{array}{l}30 \\
58 \\
47 \\
54 \\
37\end{array}$ \\
\hline $\begin{array}{l}901 \\
902 \\
903 \\
904 \\
905\end{array}$ & $\begin{array}{l}0 \\
0 \\
4 \\
0 \\
0\end{array}$ & $\begin{array}{l}4 \\
0 \\
0 \\
0 \\
4\end{array}$ & $\begin{array}{r}972 \\
1512 \\
1408 \\
1368 \\
1092\end{array}$ & $\begin{array}{l}4 \\
8 \\
8 \\
8 \\
4\end{array}$ & $\begin{array}{l}24 \\
28 \\
16 \\
16 \\
24\end{array}$ & $\begin{array}{r}79 \\
123 \\
113 \\
111 \\
89\end{array}$ & $\begin{array}{l}34 \\
55 \\
49 \\
52 \\
39\end{array}$ & $\begin{array}{l}966 \\
967 \\
968 \\
969 \\
970\end{array}$ & $\begin{array}{l}0 \\
2 \\
0 \\
0 \\
0\end{array}$ & $\begin{array}{l}0 \\
0 \\
0 \\
0 \\
4\end{array}$ & $\begin{array}{r}2304 \\
968 \\
1584 \\
1440 \\
1764\end{array}$ & $\begin{array}{r}16 \\
2 \\
48 \\
8 \\
8\end{array}$ & $\begin{array}{l}24 \\
11 \\
20 \\
24 \\
12\end{array}$ & $\begin{array}{r}185 \\
80 \\
109 \\
117 \\
143\end{array}$ & $\begin{array}{l}87 \\
35 \\
50 \\
53 \\
69\end{array}$ \\
\hline $\begin{array}{l}906 \\
907 \\
908 \\
909 \\
910\end{array}$ & $\begin{array}{l}0 \\
2 \\
0 \\
0 \\
0\end{array}$ & $\begin{array}{l}0 \\
0 \\
0 \\
0 \\
0\end{array}$ & $\begin{array}{r}1824 \\
908 \\
1368 \\
1224 \\
2016\end{array}$ & $\begin{array}{r}8 \\
2 \\
6 \\
8 \\
16\end{array}$ & $\begin{array}{r}28 \\
9 \\
30 \\
28 \\
16\end{array}$ & $\begin{array}{r}149 \\
75 \\
112 \\
99 \\
161\end{array}$ & $\begin{array}{l}68 \\
35 \\
49 \\
43 \\
77\end{array}$ & $\begin{array}{l}971 \\
972 \\
973 \\
974 \\
975\end{array}$ & $\begin{array}{l}0 \\
0 \\
4 \\
0 \\
0\end{array}$ & $\begin{array}{l}0 \\
0 \\
0 \\
0 \\
0\end{array}$ & $\begin{array}{r}972 \\
1944 \\
1120 \\
1464 \\
1680\end{array}$ & $\begin{array}{r}2 \\
54 \\
4 \\
4 \\
24\end{array}$ & $\begin{array}{l}45 \\
18 \\
12 \\
36 \\
16\end{array}$ & $\begin{array}{r}81 \\
136 \\
91 \\
121 \\
129\end{array}$ & $\begin{array}{l}26 \\
64 \\
43 \\
52 \\
57\end{array}$ \\
\hline $\begin{array}{l}911 \\
912 \\
913 \\
914 \\
915\end{array}$ & $\begin{array}{l}0 \\
0 \\
0 \\
0 \\
0\end{array}$ & $\begin{array}{l}0 \\
0 \\
0 \\
2 \\
0\end{array}$ & $\begin{array}{r}912 \\
1920 \\
1008 \\
1374 \\
1488\end{array}$ & $\begin{array}{r}2 \\
24 \\
4 \\
4 \\
8\end{array}$ & $\begin{array}{l}31 \\
16 \\
12 \\
36 \\
24\end{array}$ & $\begin{array}{r}76 \\
149 \\
83 \\
113 \\
121\end{array}$ & $\begin{array}{l}23 \\
71 \\
39 \\
48 \\
53\end{array}$ & $\begin{array}{l}976 \\
977 \\
978 \\
979 \\
980\end{array}$ & $\begin{array}{l}0 \\
0 \\
0 \\
0 \\
0\end{array}$ & $\begin{array}{l}0 \\
2 \\
0 \\
0 \\
0\end{array}$ & $\begin{array}{r}1488 \\
978 \\
1968 \\
1080 \\
2016\end{array}$ & $\begin{array}{r}12 \\
2 \\
8 \\
4 \\
48\end{array}$ & $\begin{array}{l}24 \\
20 \\
24 \\
24 \\
24\end{array}$ & $\begin{array}{r}119 \\
81 \\
161 \\
89 \\
145\end{array}$ & $\begin{array}{l}54 \\
36 \\
75 \\
37 \\
67\end{array}$ \\
\hline $\begin{array}{l}916 \\
917 \\
918 \\
919 \\
920\end{array}$ & $\begin{array}{l}0 \\
0 \\
0 \\
2 \\
0\end{array}$ & $\begin{array}{l}0 \\
0 \\
0 \\
0 \\
0\end{array}$ & $\begin{array}{r}1380 \\
1056 \\
1944 \\
920 \\
1728\end{array}$ & $\begin{array}{r}6 \\
4 \\
24 \\
2 \\
16\end{array}$ & $\begin{array}{l}20 \\
20 \\
12 \\
19 \\
40\end{array}$ & $\begin{array}{r}113 \\
87 \\
151 \\
76 \\
137\end{array}$ & $\begin{array}{l}52 \\
39 \\
73 \\
29 \\
59\end{array}$ & $\begin{array}{l}981 \\
982 \\
983 \\
984 \\
985\end{array}$ & $\begin{array}{l}0 \\
0 \\
0 \\
0 \\
0\end{array}$ & $\begin{array}{l}0 \\
0 \\
0 \\
0 \\
4\end{array}$ & $\begin{array}{r}1320 \\
1476 \\
984 \\
2016 \\
1188\end{array}$ & $\begin{array}{r}8 \\
4 \\
2 \\
16 \\
4\end{array}$ & $\begin{array}{l}24 \\
10 \\
27 \\
24 \\
24\end{array}$ & $\begin{array}{r}107 \\
122 \\
82 \\
161 \\
97\end{array}$ & $\begin{array}{l}48 \\
59 \\
28 \\
75 \\
43\end{array}$ \\
\hline $\begin{array}{l}921 \\
922 \\
923 \\
924 \\
925\end{array}$ & $\begin{array}{l}2 \\
0 \\
0 \\
0 \\
0\end{array}$ & $\begin{array}{l}0 \\
2 \\
0 \\
0 \\
4\end{array}$ & $\begin{array}{l}1232 \\
1386 \\
1008 \\
2304 \\
1140\end{array}$ & $\begin{array}{r}4 \\
4 \\
4 \\
24 \\
12\end{array}$ & $\begin{array}{l}20 \\
18 \\
30 \\
24 \\
12\end{array}$ & $\begin{array}{r}101 \\
114 \\
83 \\
181 \\
89\end{array}$ & $\begin{array}{l}46 \\
53 \\
32 \\
85 \\
42\end{array}$ & $\begin{array}{l}986 \\
987 \\
988 \\
989 \\
990\end{array}$ & $\begin{array}{l}0 \\
0 \\
0 \\
0 \\
0\end{array}$ & $\begin{array}{l}4 \\
0 \\
0 \\
0 \\
0\end{array}$ & $\begin{array}{l}1620 \\
1536 \\
1680 \\
1056 \\
2592\end{array}$ & $\begin{array}{r}8 \\
8 \\
12 \\
4 \\
32\end{array}$ & $\begin{array}{l}44 \\
24 \\
12 \\
36 \\
24\end{array}$ & $\begin{array}{r}131 \\
125 \\
135 \\
87 \\
201\end{array}$ & $\begin{array}{l}55 \\
55 \\
65 \\
35 \\
95\end{array}$ \\
\hline $\begin{array}{l}926 \\
927 \\
928 \\
929 \\
930\end{array}$ & $\begin{array}{l}0 \\
0 \\
0 \\
0 \\
0\end{array}$ & $\begin{array}{l}0 \\
0 \\
0 \\
2 \\
0\end{array}$ & $\begin{array}{r}1392 \\
1248 \\
1440 \\
930 \\
2304\end{array}$ & $\begin{array}{r}4 \\
8 \\
16 \\
2 \\
16\end{array}$ & $\begin{array}{r}40 \\
20 \\
8 \\
36 \\
24\end{array}$ & $\begin{array}{r}115 \\
101 \\
113 \\
77 \\
185\end{array}$ & $\begin{array}{l}48 \\
41 \\
55 \\
30 \\
87\end{array}$ & $\begin{array}{l}991 \\
992 \\
993 \\
994 \\
995\end{array}$ & $\begin{array}{l}2 \\
0 \\
2 \\
0 \\
0\end{array}$ & $\begin{array}{l}0 \\
0 \\
0 \\
0 \\
0\end{array}$ & $\begin{array}{r}992 \\
1536 \\
1328 \\
1728 \\
1200\end{array}$ & $\begin{array}{r}2 \\
16 \\
4 \\
8 \\
4\end{array}$ & $\begin{array}{l}17 \\
32 \\
12 \\
16 \\
24\end{array}$ & $\begin{array}{r}82 \\
121 \\
109 \\
141 \\
99\end{array}$ & $\begin{array}{l}33 \\
53 \\
52 \\
67 \\
42\end{array}$ \\
\hline $\begin{array}{l}931 \\
932 \\
933 \\
934 \\
935\end{array}$ & $\begin{array}{l}4 \\
0 \\
0 \\
0 \\
0\end{array}$ & $\begin{array}{l}0 \\
0 \\
0 \\
0 \\
0\end{array}$ & $\begin{array}{l}1120 \\
1404 \\
1248 \\
1404 \\
1296\end{array}$ & $\begin{array}{r}16 \\
6 \\
4 \\
4 \\
8\end{array}$ & $\begin{array}{l}18 \\
24 \\
16 \\
26 \\
28\end{array}$ & $\begin{array}{r}85 \\
115 \\
103 \\
116 \\
105\end{array}$ & $\begin{array}{l}37 \\
52 \\
48 \\
52 \\
39\end{array}$ & $\begin{array}{r}996 \\
997 \\
998 \\
999 \\
1000\end{array}$ & $\begin{array}{l}0 \\
2 \\
0 \\
0 \\
0\end{array}$ & $\begin{array}{l}0 \\
2 \\
0 \\
0 \\
0\end{array}$ & $\begin{array}{r}2016 \\
998 \\
1500 \\
1368 \\
1800\end{array}$ & $\begin{array}{r}12 \\
2 \\
4 \\
12 \\
40\end{array}$ & $\begin{array}{l}24 \\
14 \\
26 \\
24 \\
20\end{array}$ & $\begin{array}{r}163 \\
82 \\
124 \\
109 \\
131\end{array}$ & $\begin{array}{l}76 \\
38 \\
56 \\
43 \\
61\end{array}$ \\
\hline
\end{tabular}

\section{References}

[1] L. R. Ford, Automorphic functions, Second edition, New York (1951).

[2] R. Fricke, Lehrbuch der Algebra, Dritter Band, Braunschwieg (1928).

[3] R. Gunning, Leetures on modular forms, Princeton (1962).

[4] E. Hecke, Analytische Arithmetik der positiven quadratischen Formen, Kgl. Danske Videnskabernes Selskab. Mathematiskfysiske Meddelelser 17, 12 (1940).

[5] E. Hecke, Die Klassenzahl imaginar-quadratischer Körper in der Theorie der elliptischen Modulfunktionen. Monatshefte für Mathematik und Physik 48, 75-83 (1959).

[6] F. Klein, Gesammelte mathematische Abhandlungen, Dritter Band, Berlin (1923).

(Paper 67B1-93) 


\section{Publications of the National Bureau of Standards*}

\section{Selected Abstracts}

Tables of Einstein functions, Vibrational contributions to the thermodynamic functions, J. Hilsenrath and G. G. Zieg]er, NBS Mono. 49, (July 12, 1962) \$2.75.

Tables are presented for the contribution of a harmonic oscillator to the free energy function, enthalpy function, entropy, and heat capacity of gases. Dimensionless values of the Planck-Einstein functions are given as a function of $x=h c_{\nu} / k T$ for $x=0.0010(.0001){ }^{\circ} \mathrm{K}$ directly as a function of frequency $\nu$, and temperature $T$, was computed using the values 1.43880 for the sacond radiation constant $h c / k$, and 1.98717 for the universal gas constant $R$. The arguments for the latter table are spaced at 10 wave number intervals from 100 $\mathrm{cm}^{-1}$ to $400 \mathrm{~cm}^{-1}$.

Systems of electrical units, F. B. Silsbee, NBS Mono. 56 , (Sept. 20, 1962) 30 cents.

The various systems of measurement, with their respective sets of units, used in the literature on electricity and magnetism are described in detail. Their historical development is summarized. The manner in which each is derived from either of the two alternative points of view of the experimentalist and the theoretician is compared and contrasted. The desirability of recognizing both points of view in international standardization, particularly when discussing rationalization, is pointed out. The present status of the absolute measurements on which all electrical units are based is reported, and tables are included for the conversion of equations and numerical values from one system to another.

Tchebycheff approximations by exponentials, J. R. Rice, J. Soc. Ind. Appl. Math. 10, No. 1, 149-161 (Mar. 1962).

Tchebycheff approximations of the form

$$
\sum_{i=1}^{k} \sum_{j=0}^{m_{i}} p_{i j} x^{j} b_{j}^{x}
$$

where $\sum_{i=1}^{k}\left(m_{j}+1\right) \leq n$ are studied. With this approximating function it is shown that a best approximation on [0.1] exists for every continuous function. It is shown that this function is a varisolvent function and the uniqueness and characteristic properties of best approximations follow from known results for varisolvent functions. A theorem relating the best approximation on $[0,1]$ is established for varisolvent functions, and hence for this approximating function. The generalization to an approximating function which is an arbitrary linear combination of exponentials with polynomial coefficients is given.

Real representations of coordinate rotations, U. Fano, J. Math. Phys. 1, No. 5, 417-423 (Sept.-Oct. 1960).

Since irreducible tensorial sets that represent observables are of integral degree, their transformations under coordinate rotations have real representations. Real representations, with rows and columns classified by eigenvalues of the commuting operators $J_{z}^{2}$ and $\exp \left(i \tau J_{y}\right)$, are given explicitly, so that complex functions of rotation angles need not be used. The addition of angular momenta is worked out for sets in the real representation such as the sets of real orbital wave functions. Applications to the theory of angular distributions are discussed.

The vapor pressure of $20{ }^{\circ} \mathrm{K}$ equilibrium hydrogen, L. C. Weber, Jr., D. E. Diller, H. M. Roder, and R. D. Goodwin, cryogenics 3, 236-238 (June 1962).

The vapor pressure of $20^{\circ} \mathrm{K}$ equilibrium hydrogen has been measured between the normal boiling point and the critical point. The data are represented analytically for purposes of smoothing and interpolation and the results are compared with earlier work.

Studies in non-equilibrium rate processes. V. The relaxation of moments derived from a master equation, K. E. Shuler, K. Anderson, and G. H. Weiss, J. Math. Phys. 3, No. 3, 550-556 (May-June 1962).

A study has been made of the relaxation of the moments of probability distributions whose time evolution are governed by a master equation. The necessary and sufficient conditions for the first moment to undergo a simple exponential relaxation have been derived. The necessary and sufficient conditions under which the first $k$ moments satisfy a closed system of linear equations also have been developed. This treatment has also been extended to an investigation of the moments of the distribution determined by the Fokker-Planck equation.

Irreversible processes in liquids and the density matrix: monatomic molecules, R. E. Nettleton, Phys. Fluids 5, No. 6, 687-700 (June 1962).

A small subvolume of an infinite, isotropic, monatomic liquid is approximated by a model in which no diffusion is permitted across the boundary, and interaction with the surroundings is represented by a random force potential in the Hamiltonian. The density matrix for this model is expanded in a sum of scalar, vector, and tensor parameters, for which rate equations are derived from the quantum Liouville equation. An interpretation of these rate equations as phenomenological equations in the sense of irreversible thermodynamics leads to a set of conditions which can be used to determine the molecular configuration dependence of the density matrix and the stochastic interaction term in the Hamiltonian. The result provides a generalization, based on quantum statistics, of a previous, purely phenomenological, extension of irreversible thermodynamics to include inertial effects.

Graphs for bivariate normal probabilities, M. Zelen and N. C. Severo, Ann. Math. Stat. 31, No. 3, 619-624 (Sept. 1960). A chart is presented which enables one to quickly calculate the bivariate normal probability integral

$$
\begin{gathered}
L(h, k ; \rho)=\int_{h}^{\infty} d x \int_{k}^{\infty} g(x, y ; \rho) d y \\
\text { where } g(x, y ; \rho)=\left[2 \Pi \sqrt{1-\rho^{2}}\right]^{-1} \exp -\frac{1}{2}\left(\frac{x^{2}-2 \rho x y+y^{2}}{1-\rho^{2}}\right)
\end{gathered}
$$

The use of this chart will result in at most an error of $10^{-2}$. Examples of application are included.

Oscillatory phenomena, A. H. Kahn (Proc. Conf. High Magnetic Field, Mass. Inst. Tech., Cambridge, Mass., Nov. 1-4, 1961), Book, High Magnetic Fields, pp. 480-488 (Mass. Inst. Tech. Press, Cambridge, Mass., and John Wiley and Sons, Inc., New York, N.Y., 1962).

A quantum treatment of electrical conductivity in high magnetic fields will be presented. It will be shown how the transport coefficients and the magnetic susceptibility display variations periodic in the reciprocal of the magnetic field strength. The use of this high field behavior in the determination of electronic band structures will be discussed. The scattering of conduction electrons by impurities is greatly modified by the magnetic field. The effect on conductivity will be discussed.

Games associated with a renewal process, M. M. Siddiqui, Ann. Math. Stat. 31, 697-701 (June 1962).

Robbins (Annals of Mathematical Statistics, Vol. 32 (1961), 
pp. 187-194) considered games associated with a recurrent event, when the trials are being performed at discrete times so that the interval between two events is an integer. His results are extended to the situations where the interval between two events is not necessarily an integer, such as the interval between arrivals of two meteors within the atmosphere.

Criteria for the reality of matrix eigenvalues, M. P. Drazin and E. V. Haynesworth, Z. Math. 78, 449-452 (Mar. 1962). This note is concerned with generalizations of the known fact that an $n x n$ matrix with $n$ linearly independent eigenvectors all corresponding to real eigenvalues is similar to a hermitian matrix, and can consequently be transformed into its conjugate transpose by a hermitian similarity. For any positive integer $m$, an analogous necessary and sufficient is established for a given square complex matrix $A$ to have a set of real eigenvalues (not necessarily all distinct) to which there correspond at least $m$ linearly independent eigenvectors analogous results about eigenvalues of modulus unity and pure imaginary eigenvalues are also obtained.

The above-mentioned results are applied to establish a theorem on the reality of the eigenvalues of a certain type of matrix, and to give a new proof of a theorem of K. Goldberg but in fact Goldberg's defining conditions for the class of matrices in question are very dissimilar from ours, so the point of interest is rather the (essential) equivalence of two definitions. Another application leads to theorems about the reality of the roots of an arbitrary complex polynomial $h(\lambda)$, which refine and extend previously known theorems along similar lines.

Group theory and crystal field theory, C. M. Herzfeld and P. H. E. Meijer, Book, Solid State Physics 12, 1-91 (Academic Press, Inc., New York, N.Y., 1961).

A survey of the principal group theoretical principles and methods used in crystal field theory.

Algorithm and rapid binary division, R. S. Ledley and J. B. Wilson, IRE Trans. Electronic Computers EC-10, No. 4, 662670 (Dec. 1961).

An algorithm for performing rapid division with binary numbers is described. First a partial algorithm is presented that has the advantage of extreme simplicity, and in a majority of the cases significantly reduces the number of operations required for division. Second, the complete algorithm is given that produces the minimum number of operations for division, which will result on the average in a saving of nearly two thirds the normal division time in digital computers.

A procedure for estimating eigenvalues, N. W. Bazley and D. Fox, J. Math. Phys. 3, 469-471 (May-June 1962).

A new procedure is given for calculation of lower bounds to the eigenvalues of self-adjoint operators. Calculation of the lower bounds is reduced to the solution of linear alg $\cdot$ braic problems.

Structural and internal state variables in the description of scalar rate processes in fluids, R. E. Nettleton, Phys. Fluids 4, 1488 (1981).

Arguments are presented to show that the internal energy per molecule in a macroscopically small volume element of an infinite liquid should remain constant, in first approximation, during a sudden fluctuation in liquid structure at constant density and temperature. This result is shown to be consistent with a formulation of non-equilibrium thermodynamics in which the departures of structural parameters from their local equilibrium values appear as thermodynamic variables and in which there is no relaxing structural specific heat. However, it is shown that such a relaxing specific heat must appear in the thermodynamic treatment of thermal variables, which give the populations of internal vibrationalrotational states; and a new non-equilibrium thermodynamics of thermal relaxation, extending the earlier work of Meixner to include inertial effects, is formulated in such a way as to be consistent with this fact. The new formulation, which replaces a previous theory now shown to be valid only for structural variables, is based on the Pauli equation, generalized to include second-order time-derivatives. It is shown, on plausible assumptions about the molecular transition probabilities, that one can calculate all the rate constants and relaxation times introduced to describe inertial effects, as well as the thermodynamic forces.

Quantum-mechanical calculation of harmonic oscillator transition probabilities in a one-dimensional impulsive collision, K. E. Shuler and R. W. Zwanzig, J. Chem. Phys. 33, No. 6, 1778-1784 (Dec. 1960).

The quantum mechanical vibrational transition probabilities $P_{i \rightarrow f}(\epsilon)$ for harmonic oscillators, undergoing impulsive hard sphere collisions along the line of centers with an incident beam of atoms with relative kinetic energy $\epsilon$, have been computed by a machine (IBM-704) solution of the relevant Schrödinger equation. Curves for $P_{i \rightarrow f}(\epsilon)$ over a range of $\epsilon$ are presented for initial $(i)$ and final $(f)$ vibrational oscillator states $i, f=0,1,2$, and 3 . It is shown that this model of an inelastic collision gives rise to appreciable vibrational transitions $\nu(i) \rightarrow \nu(f)$ with $|\Delta \nu|>1$ (in addition to the $|\Delta \nu|=1$ transitions) in contrast to the Landau-Teller-Herzfeld adiabatic, 1st order perturbation treatment which permits only transitions for which $|\Delta \nu|=1$. The implication of this result is discussed in relation to the dissociation of diatomic molecules and to the adsorption of atoms on solids. Averaged transition probabilities $\bar{P}_{i \rightarrow f}(T)$ are computed for an incident beam of particles with a Maxwellian velocity distribution. It is pointed out that such averaged transition probabilities may give a misleading impression of the efficiency of translationalvibrational energy transfer if the $P_{i \rightarrow f}(\epsilon)$ show a resonance type of behavior, i.e., a strong order of magnitude dependence of $P_{i \rightarrow f}(\epsilon)$ on $\epsilon$ over a small interval of $\epsilon$.

\section{Other NBS Publications}

\section{J. Research NBS 66A (Phys. and Chem.), No. 6 (Nov.-Dec.} 1962), 70 cents.

Heat of formation of nitronium perchlorate. A. A. Gilliland. Phase equilibrium relations in the binary system bismuth sesquioxide-niobium pentoxide. R. S. Roth and J. L. Waring.

Elastic constants of rutile $\left(\mathrm{TiO}_{2}\right)$. J. B. Wachtman, Jr. W. E. Tefft, and D. G. Lam, Jr.

Reaction of hardened portland cement paste with carbon dioxide. C. M. Hunt and L. A. Tomes.

Titanium standards for hydrogen content. J. T. Sterling, F. J. Palumbo, and L. L. Wyman.

Ultraviolet stability of crosslinked polycaprolactam systems. S. D. Bruck.

Spectral-line intensities and $g f$-values in the first spectrum of copper. C. H. Corliss.

Batch adsorption from solution. W. V. Loebenstein.

Separation of hafnium from zirconium and their determination: Separation by anion-exchange. L. A. Machlan and J. L. Hague.

J. Research NBS 67 A (Phys. and Chem.), No. 1 (Jan.-Feb. 1963), 70 cents.

Heat of formation of calcium aluminate monosulfate at $25{ }^{\circ} \mathrm{C}$. H. A. Berman and E. S. Newman.

2,3-Dimethylpentane and 2-methylhexane as a test mixture for evaluating highly efficient fractionating columns. E. C. Kuehner.

Phase equilibrium relations in the $\mathrm{Sc}_{2} \mathrm{O}_{3}-\mathrm{Ga}_{2} \mathrm{O}_{3}$ system. S. J. Schneider and J. Li Waring.

Analysis of two infrared bands of $\mathrm{CH}_{2} \mathrm{D}_{2}$. W. B. Olson, H. C. Allen, Jr., and E. K. Plyler.

Precise coulometric titrations of halides. G. Marinenko and J. K. Taylor.

Radial distribution study of vitreous barium borosilicate. G. J. Piermarini and S. Block.

Dynamic compressibility of poly (vinyl acetate) and its relation to free volume. J. E. McKinney and H. V. Belcher. An investigation of the constitution of the mercury-tin system. D. F. Taylor and C. L. Burns.

Effect of methyl bromide additions on the flame speed of methane. C. Halpern. 
J. Research NBS $67 \mathrm{C}$ (Eng. and Instr.), No. 1 (Jan.-Mar. 1963), 75 cents.

Method for calibrating a standard volt box. B. L. Dunfee. Stability of residual thiosulfate in processed microfilm. C. I. Pope.

Equipment for single crystal growth from aqueous solution. J. L. Torgesen, A. T. Horton, and C. P. Saylor.

An automatic multichannel correlator. R. F. Brown, Jr.

Elastic constant-porosity relations for polycrystalline thoria.

S. Spinner, F. P. Knudsen, and L. Stone.

An oxygen partial pressure warning instrument. Greenspan.

New fast-opening, large-aperture shutter for high-speed photography. E. C. Cassidy and D. H. Tsai.

Equations for the radiofrequency magnetic permeameter. C. A. Hoer and A. L. Rasmussen.

J. Research NBS 67D (Radio Prop.), No. 1 (Jan.-Feb. 1963), 70 cents.

A lunar theory reasserted - a rebuttal. J. V. Evans.

Point-to-point communication on the moon. L. E. Vogler.

HF communication during ionospheric storms. G. E. Hill.

Use of surface refractivity in the empirical prediction of total atmospheric refraction. W. R. Iliff and J. M. Holt.

Effective sunspot numbers. W. B. Chadwick.

On the theory of radio wave propagation over inhomogeneous earth. K. Furutsu.

Fields of electric dipoles in sea water (a correction). W. Anderson.

Composition of reflection and transmission formulae. J. Heading.

Titheridge coefficients for the polynomial method of deducing electron density profiles from ionograms. A. R. Long and J. O. Thomas.

Input admittance of linear antennas driven from a coaxial line. T. T. Wu.

Bibliography on atomic transition probabilities, B. M. Glennon and W. L. Wiese, NBS Mono. 50 (Aug. 1, 1962), 35 cents.

Experimental transition probabilities for spectral lines of seventy elements, C. H. Corliss and W. R. Bozman, NBS Mono. 53 (July 20, 1962), \$4.25.

Chemistry of cement. Proceedings of the fourth international symposium, Washington, 1960, NBS Mono. 43, Vol. I (Aug. 31, 1962), \$5.75; Vol. II (Sept. 27, 1962), $\$ 5.50$. The two volumes are available at $\$ 11.25$ a set.

Quarterly radio noise data, March, April, May 1962 and corrigendum for Technical Notes 18-1 through 18-11, W. Q. Crichlow, R. T. Disney, and M. A. Jenkins, NBS Tech. Note 18-14, (August 9, 1962) 50 cents.

Mean electron density variations of the quiet ionosphere, No. 8 -October 1959 , J. W. Wright, L. R. Wescott and D. J. Brown, NBS Tech. Note 40-8, (September 1962) 35 cents.

Synoptic radio metrology, B. R. Bean, J. D. Horn, and L. P. Riggs, NBS Tech. Note 98, (October 1962) 50 cents.

A survey of the techniques for measuring the radio refractive index, R. E. McGavin, NBS Tech. Note 99 (May 1962), 30 cents.

Required signal-to-noise ratios, RF signal power, and bandwidth for multichannel radio communications systems, E. F. Florman and J. J. Tary, NBS Tech. Note 100 (Jan. $1962), \$ 1.00$.

A tabulation of the thermodynamic properties of normal hydrogen from low temperatures to $540^{\circ} \mathrm{R}$ from 10 to 1500 psia, Supplement A (British units), J. W. Dean, NBS Tech. Note 120 A (June 1962) 45 cents.

Bibliography on direction finding and related ionospheric propagation topics, 1955-1961, O. D. Remmler, NBS Tech. Note 127, (Oct. 1962) 60 cents.

Controlled temperature oil baths for saturated standard cells, P. H. Lowrie, Jr., NBS Tech. Note 141, (Aug. 1962) 25 cents.

Equatorial spread F, W. Calvert, NBS Tech. Note 145 (Aug. 1, 1962) 60 cents.

General characteristics of linear strain gage accelerometers used in telemetry, P. S. Lederer, NBS Tech. Note 150 (June 1962) 40 cents.
Mode conversion in the earth-ionosphere waveguide, J. R Wait, NBS Tech. Note 151 (June 8, 1962) 20 cents.

A general survey of the semiconductor field, G. W. Reimherr, NBS Tech. Note 153 (Aug. 1962) 30 cents.

The thermodynamic properties of helium from 3 to $300^{\circ} \mathrm{K}$ between 0.5 and 100 atmospheres, D. B. Mann, NBS Tech. Note 154 (Jan. 1962) 50 cents.

The energy parameter B for strong blast waves, D. L. Jones, NBS Tech. Note 155, (July 1962) 25 cents.

Information selection systems retrieving replica copies: A state-of-the-art report, T. C. Bagg and M. E. Stevens, NBS Tech. Note 157 (Dec. 31, 1961) $\$ 1.25$.

Efficient use of the radio spectrum, K. A. Norton, NBS Tech. Note 158 (Apr. 1962) 45 cents.

A Fortran code for calculation of eigenvalues and eigenfunctions in real potential wells, R. S. Caswell, NBS Tech. Note 159 (Aug. 1962) 25 cents.

Evaluation of unexpectedly large radiation exposures by means of photographic film, W. L. McLaughlin, NBS Tech. Note 161, (Aug. 1962) 15 cents.

Photosensitized reaction between hydrogen $(2 \mathrm{p})$ rho atoms and molecular nitrogen, I. Tanaka and J. R. McNesby, J. Chem. Phys. 36, No. 12, 3170-3173 (June 15, 1962).

Effect of particle size on low-temperature heat capacities, A. C. Victor, J. Chem. Phys. 36, No. 10, 2812-2813 (May $15,1962)$

Physical standards of emittance and reflectance, J. C. Richmond, (Proc. Conf. Radiative Transfer from Solid Materials, Boston, Mass., Dec. 12-13, 1960), Book, Radiative Transfer from Solid Materials, ed. H. H. Blau, Jr., and H. Fischer, Sec. III, pp. 142-153 (The Macmillan Co. New York, N.Y., 1962).

Effect of molecular oxygen on the emission spectra of atomic oxygen-acetylene flames, S. L. N. G. Krishnamachari and H. P. Broida, J. Chem. Phys. 34, 1709-1711 (1961).

Signs of nuclear resonance coupling constants in saturated aliphatic systems, H. Finegold, Proc. Chem. Soc. pp. 213-214 (June 1962).

The moropholy of mid-latitude 6300 angstrom ares, T. Tohmatsu and F. E. Roach, J. Geophys. Res. 67, No. 5 , 1817-1821 (May 1962).

Some electrical properties of the porous graphite contact on $p$-type silicon, G. G. Harman, T. H. Higier, and O. L Meyer, J. Appl. Phys. 33, 2206 (July 1962).

The structure of the vibrational-rotational bands of an asymmetric rotor, H. C. Allen, Jr., Phil. Trans. Roy. Soc London, Ser. B. Math. and Phys. Sci. 253, No. 1030 , 335-357 (Apr. 27, 1961).

A combined analog-digital differential analyzer (CADDA) W. D. Urban, W. R. Hahn, Jr., and H. K. Skramstad, Proc. Combined Analog Digital Computer Systems Symp., Phila., Pa., Dec. 1960, 2d item (1960).

The types of blackout, their time variations, and the mechanisms producing them, V. Agy, J. Phys. Soc. Japan 1', 93-97 (Sept. 1961).

Some properties of dirty contacts on semi-conductors and resistivity measurements by a two terminal method, G. G. Harman and T. Higier, J. Appl. Phys, 33, 2198 (July 1962).

A property of linear frequency modulation, A. J. Goldman, Proc. IRE 50, No. 7, 1711 (July 1962).

The vapor pressure of $20^{\circ} \mathrm{K}$ equilibrium hydrogen, L. C Weber, Jr., D. E. Diller, H. M. Roder, and R. D. Goodwin, Cyrogenic 3, 236-238 (June 1962).

The thermal properties of powder insulators in the temperature range $300^{\circ}-4^{\circ} \mathrm{K}, \mathrm{D}$. Cline and $\mathrm{R}$. H. Kropschot (Proc. Conf. Radiative Transfer from Solid Materials, Boston, Mass., Dec. 12-13, 1960), Book, Radiative Transfer from Solid Materials, ed. H. H. Blau, Jr., and H. Fischer, sec. I, pp. 61-81 (The Macmillan Co., New York, N.Y., 1962)

Kinetics of the hydrolysis of acetal in $N$-methylpropionamidewater and $N, n$-dimethylformamide-water solvents at 20 25, 30, and $40^{\circ}$, R. K. Wolford and R. G. Bates, J. Phys. Chem. 66, No. 8, 1496-1500 (1962).

Symposium on spectroscopic excitation, B. F. Scribner, Am. Soc. Testing Materials Spec. Tech. Publ. 259, 1 (Oct. 1960).

Path loss measurements versus prediction for long distance tropospheric scatter circuits, A. F. Barghausen and C. F. 
Peterson, IRE Trans. Commun. Systems CS-9, No. 4, 439-445 (Dec. 1961).

Introduction to the theory of V.L.F. propagation, J. R Wait, Proc. IRE 50, 1624-1647 (July 1962).

Some experimental aspects of nuclear orientation, E. Ambler, Proc. Tenth Intern. Congress of Refrigeration, Copenhagen, Denmark, I, 195-198 (1959)

The densities of saturated liquid hydrogen, R. D. Goodwin, D. E. Diller, H. M. Roder, and L. A. Weber, Cryogenics 2, 81-83 (Dec. 1961).

Reference tables for $40 \%$ iridium- $60 \%$ rhodium versus iridium thermocouples, G. F. Blackburn and F. R. Caldwell, Book, Temperature, Its Measurement and Control in Science and Industry 3, Pt. 2, 161-175 (Reinhold Publ. Corp., New York, N.Y., 1962).

The cement reference laboratory (1929-1959), J. R. Dise, Am. Soc. Testing Materials Proc. 59, 369 (1959).

International coordination of measurement, A. G. MeNish, Sci. Math. Weekly 2, No. 3, 28-29 \& 35 (Sept. 1961).

The calibration at the National Bureau of Standards of mass standards for ultramicroanalysis, L. B. Macurdy, Book, Vacuum Microbalance Techniques, ed. R. F. Walker, 2, 165-175 (Plenum Press, Inc., New York, N. Y., 1962).

Post office mechanization, B. M. Levin, M. C. Stark and P. C. Tosini, Elec. Eng. 80, No. 2, 105-110 (Feb. 1961).

Switching properties in ferroelectrics of the family $\mathrm{Bi}_{4} \mathrm{Ba}_{m-2}$ $\mathrm{Ti}_{m+1} \mathrm{O}_{?(m+2)}$, P. H. Fang and E. Fatuzzo, J. Phys. Soc. Japan 17, 238 (1962).

Rate of vaporization of refractory substances, J. J. Diamond, J. Efimenko, R. F. Hampson, and R. F. Walker (Proc. 4th Intern. Symp. Reactivity of Solids), Book, Reactivity of Solids, ed. J. H. de Bower, et al., p. 725 (Elsevier Publ. Co., Amsterdam, The Netherlands, 1961).

A derivation of the relaxation spectrum representation of the mechanical response function, R. S. Marvin, Repts. Progr. Polymer Phys. Japan 5, 56-58 (1962).

Thermal isomerization of isopropyl-1,1,1-d radicals, W. M. Jackson and J. R. MeNesby, J. Chem. Phys. 26, No. 9, 2272-2275 (May 1, 1962).

Research on light metals in the Metallurgy Division, National Bureau of Standards, T. G. Digges, (Symp. Light Metal Industry, Jamshedpur, India) Indian Construction News, p. 68 (Aug. 1961).

A nomograph for selecting light balancing filters for camera exposure of color films, C. S. McCamy, Med Biol. Illustration, London, England 11, No. 1, 13-15 (Jan. 1961).

Young's modulus of single crystal corundum from $77^{\circ} \mathrm{K}$ to $850^{\circ} \mathrm{K}$, J. B. Wachtman, Jr., W. E. Tefft, and D. G. Lam, Jr., Book, Mechanical Properties of Engineering Ceramics, pp. 221-223 (Interscience Publ. Inc., New York, N. Y., 1961).

Attempts to eliminate fatigue damage by heat treatment, J. A. Bennett, Am. Soc. Metals, Trans. Quart. (Tech. Notes) 55, No. 2, 362-363 (June 1962).

A controller for maintaining a constant rate of vaporization in fractional distillation, E. C. Kuehner and R. T. Leslie, Anal. Chem. 34, No. 9, 1155-1156 (Aug. 1962).

Nuclear moment of NI ${ }^{61}$, L. H. Bennett and R. L. Streever, Jr., Phys. Rev. Letters 121, No. 6, 2141-2142 (June 15, 1962).

A method for determining mechanical resonance frequencies and for calculating elastic moduli from these frequencies, S. Spinner and W. E. Tefft, Am. Soc. Testing Materials Proc. 61, 1221-1238 (1961).

New uses of microfilm with electronic scanners, a progress report on FOSDIC III, M. L. Greenough, Proc. 8th Annual Meeting Natl. Microfilm Assoc., Wash., D.C., Apr. 2-4, 1959, p. 278 (Annapolis, Md., 1959).

Susceptor elements for high temperature induction heating, S. Hasko and H. S. Parker, Am. Ceram. Soc. Bull. 41, No. 7, 467 (July 1962).

The dynamic compressibility of a rubber-sulfur vulcanizate and its relation to free volume, J. E. McKinney, H. V. Belcher, and R. S. Marvin, Trans. Soc. Rheology 4, 347-362 (1960).

Relation of solar active regions at central meridian passage to ionospheric disturbance, C. S. Warwick and J. V. Lincoln, AGARDograph Proc. Sixth AGARD Ionospheric Research Committee Meeting (Rome, Italy, May 15-18, 1961).
Suggested arrangement of mirrors to form multiple reference angles, J. B. Saunders, J. Opt. Soc. Am. 51, No. 8, 859-862 (Aug. 1961).

Radiolysis of ethane-1,1,1- $d_{2}$, L. J. Stief and P. Ausloos, J. Chem. Phys. 36, No. 11, 2904 (June 1, 1962).

D-C differential current meter, E. Niesen, Rev. Sci. Instr. 32, No. 12, 1407-1408 (Dec. 1961).

Radiowave propagation during World War II, K. L. Norton, Proc. IRE 50, No. 5, 698-704 (May 1962).

Development directions to keep leather apace, J. R. Kanagy, Leather and Shoes 142, 34-35 (Aug. 12, 1961).

High-energy X-ray spectrometer using large anticoincidence sodium iodide crystals, J. M. Wycoff, Proc. Total Absorption Gamma-Ray Spectrometry Symp., Gatlinburg, Tenn., May 10, 1960, pp. 201-210 (1960).

Radio propagation quality in the North Atlantic area 19531960, M. E. Nason, AGARDograph Proc. 6th AGARD Ionospheric Research Committee Meeting (Rome, Italy, May 15-18, 1961).

Discussion of Feldman's and Sereda's Paper "Characteristics of sorption and expansion isotherms of reactive limestone aggregate," C. M. Hunt, L. A. Tomes, R. G. Pike, and D. Hubbard, J. Am. Concrete Inst. 59, 815-818 (Mar. 1962).

The National Bureau of Standards Library, S. A. Jones, D.C. Libraries 32, 7-10 (Jan. 1961).

Systematic errors, W. J. Youden, Ordnance XLVI, No. 248, 299-301 (Sept.-Oct. 1961).

Determination of the dissociation equilibria of water by a conductance method, H. C. Duecker and W. Haller, J. Phys. Chem. 66, No. 2, 225-229 (Feb. 1962).

Heat of formation of the most stable form of metaboric acid, $\mathrm{HBO}_{2}(\mathrm{cI})$, M. V. Kilday and E. J. Prosen, J. Am. Chem. Soc. Commun. to Editor 82, 550 (1960).

The scientific contributions of William Weber Coblentz, E. K. Plyler, J. Appl. Spect. 16, No. 3, 73-77 (1962).

Absorption spectrum of $\mathrm{CF}_{2}$ trapped in an argon matrix, A. M. Bass and D. E. Mann, J. Chem. Phys. 36, No. 12, 3501-3502 (June 15, 1962)

The rapid selector and other NBS document retrieval studies, J. L. Pike and T. C. Bagg, Proc. National Microfilm Assoc., Annual Meeting, Wash., D.C., Apr. 25-27, 1962, XI, 213-227 (Annapolis, Md., 1962).

A test method for air-entrainment of standard Ottawa sand, M. R. DeFore and H. J. Corah, ASTM Bull. No. 248, 48-56 (TP162-166) (Sept. 1960).

The angular distribution of beta particles from oriented cerium-141 nuclei, D. D. Hoppes, Proc. Seventh Intern. Conf. Low Temperature Physics, University of Toronto, Canada, Aug. 1960, p. 186 (University of Toronto Press, Toronto, Canada, 1960).

Die radiometeorologie and ihre bedeutung fur die ausbreitung der $\mathrm{m}-, \mathrm{dm}-$ and $\mathrm{cm}-$ wallen auf grosse entfernungen, B. R. Bean, L. Fehlhaber, and J. Grosskopf, Nachr. Z (NTZ), p. 9-16 (Jan. 1962).

The program at the National Bureau of Standards, L. M. Branscomb, Book, Optical Spectroscopic Measurements of High Temperatures, ed. P. J. Dickerman, p. 235 (Univ. of Chicago Press, Chicago, Ill. 1961).

Determination of oxidation rates of air-blown asphalts by infrared spectroscopy, J. R. Wright and P. G. Campbell, J. Appl. Chem. 12, 256-266 (June 1962).

Thermocouple materials, F. R. Caldwell, Temp. Meas. Control. Sci. Ind. 3, pt. 2, 81-134 (Reinhold Publ., Corp., New York, N.Y., 1962).

Study of "valley problem" with a ray tracing program, K. Davies and A. K. Saha, Electron Density Profiles, pp. $162-166(1962)$

The extrapolation of the orthorhombic $N$-paraffin melting properties to very long chain lengths, M. G. Broadhurst, J. Chem. Phys. 36, No. 10, 2578 (May 15, 1962).

An equation of state for calculating the thermodynamic properties of helium at low temperatures, R. D. McCarty and R. B. Stewart, Book, Progress in International Research on Thermodynamic and Transport Properties, pp. 107-117 (Academic Press, Inc., New York, N.Y., 1962).

Thermal expansion of some engineering materials from $20^{\circ} \mathrm{K}$ to $293^{\circ} \mathrm{K}, \mathrm{V}$. Arp, J. H. Wilson, L. Winrich, and P. Sikora, Cryogenics 3, 230 (June 1962).

Interlaboratory evaluation of a method for indicated bright- 
ness of papers containing fluorescent brighteners, T. W. Lashof and J. M. Patek, Tappi 45, No. 7, 566 (July 1962).

An intermediate size automatically controlled hydrogen refrigeration system, D. B. Chelton, D. B. Mann, and B.W. Birmingham, Suppl. Bull. Inst. Intern du Froid, Eindhoven, Comm. 1, Annexe 1960-61, 169-178 (1960).

Thermal radiation standards and measurements of the Radiometry Section at the National Bureau of Standards, A. G. Maki, (Proc. Conf. Radiative Transfer From Solid Materials, Boston, Mass., Dec. 12-13, 1960), Book, Radiative Transfer from Solid Materials, ed. H. H. Blau, Jr., and H. Fischer, Sec. III, pp. 135-141 (The Macmillan Co., New York, N. Y., 1962).

Absolute microwave refractometer, M. J. Vetter and M. C. Thompson, Jr., Rev. Sci. Instr. 33, 656-660 (June 1962).

The information problem in government, S. N. Alexander, Proc. Engineering Information Symp. (Sponsored by the Engineers Joint Council), pp. 15-16 (Jan. 17, 1962).

Problems in the temperature calibration of an X-ray diffractometer furnace, F. A. Mauer and L. H. Bolz, (Proc. Tenth Annual Conf. Applications of X-ray Analysis, Denver, Colo., Aug. 7-9, 1961), Book, Advances in X-ray analysis, p. 229 (Plenum Press, Inc., New York, N.Y., 1962).

The detection and study of solar cosmic ravs by radio techniques, D. K. Bailey, (Intern. Conf. on Cosmic Rays and the Earth Storm), J. Phys. Soc. Japan 17, Suppl. A-1, pt. I, 106-112 (1962).

Precision and accuracy-experiment design aspects, C Eisenhart, Conf. on Applications of Statistical Methods in the Chemical Industry, Feb. 4, 1961, (Sponsored by Am. Soc. for Quality Control, Metropolitan Section, Seaton Hall University, South Orange, N.J.), p. 1 (1961).

Purification and vapor pressure of pure nitric oxide, E. E. Hughes, J. Chem. Phys. Letter to Editor 35, No. 4, 15311532 (Oct. 1961).

Ultrasounds induce flaking of ceramics from metals, J. W. Pitts, Metal Progr. 82, No. 2, 114-115 (Aug. 1962).

Ferroelectricity in the compound $\mathrm{Ba}_{2} \mathrm{Bi}_{4} \mathrm{Ti}_{5} \mathrm{O}_{18}, \mathrm{P}$. H. Fang and B. Aurivillius, Phys. Rev. 126, No. 3, 893 (May 1962).

The ammonia beam maser as a standard of frequency, J. A Barnes, D. W. Allan, and A. E. Wainwright, IRE Trans. Instrumentation I-11, 26-30 (June 1962)

Use of gas phase chromatography for rapid determination of carbonate at low levels, F. G. Carpenter, Anal. Chem. 34, 66 (Jan. 1962).

An experimental investigation of the scintillation of radio stars observed at frequencies of 223 and 456 megacycles per second from a location close to the auroral zone, C. G. Little, G. C. Reid, E. Stiltner, and R. P. Merritt, J Geophys. Res. 67, No. 5, 1763-1784 (May 1962).

The radiative formation and destruction of negative ions, L. M. Branscomb, Proc. Fifth Intern. Conf. Ionization Phenomena in Gases, Munich, Germany, I, 1-18 (North Holland Publ. Co., Amsterdam, The Netherlands, 1961).

A computer for weather data acquisition, P. Meissner, J. A. Cunningham, and C. A. Kettering, Proc. Eastern Joint Computer Conf., Dec. 13-15, 1960, p. 57 (New York, N.Y., 1960)

Position titles of chemists, W. K. Wilson, Capital Chemist 10, 145 (May 1960)

Corrected calculations of sound absorption in non-associated liquids, R. E. Nettleton, J. Acoust. Soc. Am. 34, No. 3, 350 (Mar. 1962)

International comparisons of radioactivity standards, W. E., Perry and W. B. Mann, Proc, Ninth Intern. Congress on Radiology, Munich, Germany, July 1959, 23, VII-30. VII, 1338-1342 (Urban and Schwarzenberg, Munich, Germany, 1960).

A rapid simple method of estimating the order of chemical reactions, J. H. Flynn, Chem. Eng. 69, No. 17, 137-104 (Aug. 20, 1962).

Paramagnetic resonance phenomena, H. E. Radford, Encyclopaedic Dictionary of Physics 5, 293 (Pergamon Press Inc., New York, N.Y., 1962).

Chilled-air-distribution in refrigerated trailers, P. R. Achenbach, Suppl. Bull. Intern. Inst. of Refrigeration, p. 9 (19611962).

Mass spectrum and appearance potentials of tetrafluorohy- drazine, J. T. Herron and V. H. Dibeler, J. Chem. Phys. 33, No. 5. 1595-1596 (Nov. 1960).

Large longitudinal retarded elastic deformation of rubberlike network polymers, H. Leaderman, J. Polymer Sci. 59, No. 168, S42 (June 1962).

A survey of polar cap absorption events (solar proton events) in the period 1952 through 1960, D. K. Bailey and J. M. Harrington, (Intern. Conf. on Cosmic Rays and the Earth Storm), J. Phys. Soc. Japan 17, Supply. A-II, pt. II, 334337 (1962).

Magnifications of a telescope, R. E. Stephens, J. Opt. Soc. Am. 51, No. 7, 803-804 (July 1961)

Isotope effect in an electric field and jump frequencies for diffusion in ionic crystals, J. R. Manning, J. Appl. Phys. 33, No. 7, 2145-2151 (July 1962).

Determination of isotope effects by "double labeling" oxidation of $d$-glucose with iodine, H. S. Isbell, L. T. Sniegoski, and H. L. Frush, Anal. Chem. 34, No. 8, 982-984 (July 1962).

Methods of nuclear orientation, E. Ambler, Book, Progress in Cryogenics, ed. K. Mendelssohn III, 235-280 (Heywood and Co., Ltd., London, England, 1960).

Linear thermal expansion of aluminum oxide and thorium oxide from $100^{\circ}$ to $1100^{\circ}$ K., J. B. Wachtman, Jr., T. G. Scuderi, and G. W. Cleek, J. Am. Ceram. Soc. 45, 310-323 (July 1962)

Molecular collision models and transition probabilities in liquids, R. E. Nettleton, J. Chem. Phys. 38, 2226-2227 (Apr. 1962)

Electronic development and production in the USSR, C. P. Marsden, Encylcopedia on Russia and the Soviet Union, pp. 158-160 (McGraw-Hill Book Co., Inc., New York, N.Y., 1961)

Microwave spectrum of trimethylarsine, D. R. Lide, Jr., Spectrochem. Acta 15, 473 (1959).

Lattice frequencies and rotational barriers for inorganic carbonates and nitrates, R. A. Schroeder, C. E. Weir, and E. R. Lippincott, J. Chem. Phys. 36, No. 10, 2803-2804 (May 1962)

Ferroelectricity in the compound $\mathrm{Bi}_{4} \mathrm{Ti}_{3} \mathrm{O}_{12}, \mathrm{P}$. H. Fang and C. R. Robbins, Phys. Rev. 126, No. 3, 892 (May 1962).

Environmental characteristics of a small underground fallout shelter, P. R. Achenbach, F. J. J. Drapeau, and C. W. Phillips, ASHRAE J. 4, No. 1, 21 (Jan. 1962).

Opportunities in dental research, G. C. Paffenbarger, J. Am. Dental Assoc. 60, No. 3, 413-414 (Mar. 1960).

On the nature of equatorial slant sporadic E, R. Cohen, K. L. Bowles and W. Calvert, J. Geophys. Res. 6\%, No. 3, 965972 (Mar. 1962)

Elementary statistical design, W. J. Youden, Conf. on Applications of Statistical Methods in the Chemical Industry, Feb. 4, 1961, (Sponsored by Am. Soc. for Quality Control, Metropolitan Section, Seaton Hall University, South Orange, N.J.), p. 43 (1961).

Dielectric loss in "non-polar" polymers, A. J. Curtis, J. Chem. Phys. 36, No. 12, 3500 (June 15, 1962).

Mobilities of positive ions in argon, E. C. Beaty, Proc, Conf. Ionization Phenomena in Gases, Aug. 28-Sept. 1. 1961, Munich, Germany, p. 183 (North Holland Publ. Col., Amsterdam, The Netherlands, 1962).

Blue-glass filters to approximate the blackbody at $6500^{\circ} \mathrm{K}$, D. B. Judd, Farbe 10, No. 1-4, 31 (1961).

Correction to "doppler studies of the ionosphere with vertical incidence," K. Davies, Proc. IRE 50, No. 6, 94 (June 1962).

Electron photodetachment from ions and elastic collision cross sections for O, C, Cl and F, J. W. Cooper and J. B. Martin, Phys. Rev. 126, No. 4, 1482 (May 15, 1962).

The following articles were presented before the 1961 Cryogenic Engineering Conference and published in the Book, Advances in Cryogenic Engineering $\boldsymbol{\gamma}$ (Plenum Press, Inc., New York, N.Y., 1962):

A cryostat for tensile tests in the temperature range $300^{\circ}$ to $4^{\circ}$ K, R. P. Reed, Paper K-3, 448-454.

A method of measuring shear modulus from $-424^{\circ}$ to $70^{\circ} \mathrm{F}$, R. P. Mikesell and R. M. MeClintock, Paper K-11509513.

An electrically controlled guarded flat plate calorimeter, D. Cline and R. H. Kropschot, Paper L-5, 534-538.

An experimental investigation of the individual boiling and 
condensing heat transfer coefficients for hydrogen, D. E. Drayer and K. D. Timmerhaus, Paper J-6, 401-412.

Dry gas operation of ball bearings at cryogenic temperatures, L. E. Scott, D. B. Chelton, and J. A. Brennan, Paper G-3, $273-276$

Low temperature characteristics of some commercial thermocouples, R. L. Powell and L. P. Caywood, Jr., Paper L-2, $517-521$.

Martensitic transformation products and mechanical properties of austenitic stainless steels at low temperatures, C. J. Guntner and R. P. Reed, Paper K-9, 500-502.

On the bulk density of boiling liquid oxygen, R. W. Arnett, Paper F-4, 214-218.

Temperature dependence of magnetic losses, J. J. Gniewek and R. L. Powell, Paper H-1, 303-310.

Testing of ball bearings with five different separator materials at 9200 RPM in liquid nitrogen, J. A. Brennan, W. A. Wilson, R. Radebaugh, and B. W. Birmingham, Paper $\mathrm{G}-2,262-272$.

The application and behavior of elastomers at cryogenic temperatures, R. F. Robbins, D. H. Weitzel, and R. N. Herring, Paper H-6, 343-352.

The application of gas-lubricated bearings to a miniature helium expansion turbine, B. W. Birmingham, H. Sixsmith, and W. A. Wilson, Paper A-4, 30-42.

The efficiency of an ideal refrigerator, R. B. Jacobs, Paper L-11, 567-571.

Application of reflectometer techniques to accurate reflection measurements in coaxial systems, R. W. Beatty and W. J. Anson, Proc. Inst. Elec. Engrs. B109, 345-348 (July 1962).

Chemically induced vibrational excitation: Hydroxyl radical emission in the 1-3-micron region produced by the $\mathrm{H}+\mathrm{O}_{3}$ atomic flame, D. Garvin, H. P. Broida, and H. J. Kostkowski, J. Chem. Phys. 37, No. 1, 193 (July 1962).

Gas-phase radiolysis of propane, P. Ausloos and S. Lias, J. Chem. Phys. 36, No. 12, 3163-3170 (July 15, 1962).

Four methods for predicting the durability of roofing asphalts, S. H. Greenfeld and J. R. Wright, Mater. Res. Std. 2, No. 9, 738-745 (Sept. 1962).

The absolute simplest form in digital circuit design, R. S. Ledley, Book, Digital Computers and Control Engineering, 835 pgs., (McGraw-Hill Book Co., Inc., New York, N.Y., $1960)$

Infrared spectrum of dideuteroacetylene $\left(\mathrm{C}_{2} \mathrm{D}_{2}\right), \mathrm{E} . \mathrm{D}$. Tidwell and E. K. Plyler, J. Opt. Soc. Am. 52, No. 6, 656-664 (June 1962)

Coring an electroform, W. H. Metzer, Jr., Plating 49, No. 8, 880 (Aug. 1962).

The standards challenge, A. H. Scott, Insulation 8, No. 2, 48-50 (Feb. 1962)

A system for generating "pronounceable" names using a computer, A. L. Leiner and W. W. Youden, J. Assoc. Computing Mach. 8, No. 1, 97-103 (Jan. 1961).

Using digital computers in the design and maintenance of new computers, A. L. Leiner, A. Weinberger, H. Loberman, and C. Coleman, IRE Trans. Electronic Computers $\mathbf{E C}-\mathbf{1 0}$, No. 4, 680-690 (Dec. 1961)

Surface preparation of solid metallic samples for X-ray spectrochemical analysis, R. E. Michaelis and B. A. Kilday, Book, Advances in X-ray Analysis 5, 405-411 (Plenum Press, Inc., New York, N.Y., 1962).

Infrared wavelength dependence of the total absorptivity of electroplated silver, D. Cline, J. Appl. Phys. 33, 2310-2311. (July 1962)

Simple stain gauge-based load controller, L. Mordfin and R. L. Bloss, Rev. Sci. Instr. 33, No. 7, 772-773 (July 1962).

Quantum-mechanical calculation of harmonic oscillator transition probabilities. II. Three-dimensional impulsive collisions, F. H. Mies and K. E. Shuler, J. Chem. Phys. 3\%, No. 1, 177-181 (July 1, 1962).

Remote phase control of station WWV, R. F. Fey, J. B. Milton, and A. H. Morgan, Nature 193, No. 4820, 10631064 (Mar. 17, 1962).

Melting and contractility of feather keratin, L. Mandelkern, J. C. Halpin, and A. F. Diorio, J. Polymer Sci. Letter to Editor 60, No. 169, S31-S33 (July 1962).

Wave propagation around a curved boundary which contains an obstacle, J. R. Wait, Can. J. Phys. 40, 1010-1016 (Aug. 1962).
Four-place table decibels return loss to magnitude of voltage reflection coefficient, R. W. Beatty, Microwave Eng. Handb. \& Buyers Guide, pp. TD188-192 (Nov. 1961).

Duration and spacing of sferic pulses, R. F. Linfield and C. A. Samson, Proc. IRE 50, 1841-1842 (Aug. 1962).

$\mathrm{VHF}$ and UHF signal characteristics observed on a long knife-edge, A. P. Barsis and R. S. Kirby, IRE Natl. Conv. Record, pp. 17-34 (1961).

Some observations on growing crystals of argon, L. H. Bolz, H. P. Broida, and H. S. Peiser, Acta Cryst. 15, No. 8, 810-812 (Aug. 1962).

Methods and techniques of low and very low frequency monitoring at Boulder Laboratories, A. H. Morgan and D. H. Andrews, Consultative Committee for Definition of the Second Intern. Comm. on Weights and Measures Apr. 11-12, 1961, pp. 1-10 (June 1961).

Insulation resistance measurements, A. H. Scott, Proc. 4th Electrical Insulation Conf., Materials and Application, Wash., D.C., p. 115-117 (Feb. 19-22, 1962)

Physical measurement: Pilot of progress, A. V. Astin, Steel, p. 112 (July 23, 1962).

Some tensile properties of amalgam, M. S. Rodriguez and G. Dickson, J. Dental Res. 41, No. 4, 840-852 (JulyAug. 1962)

Vacuum photolysis of solid ethane at $77^{\circ}$ K, M. D. Scheer, J. McNesby, and R. Klein, J. Chem. Phys. 36, No. 12, 3504-3505 (June 1962).

Geomagnetic and solar data, J. V. Lincoln, J. Geophys. Res. 67, No. 5, 2035-2038 (May 1962).

A study of the chemical and physical properties of magnetic recording tape, F. Nesh and R. F. Brown, Jr., IRE Trans. Audio AU-10, No. 3, 70-71 (May-June 1962).

Supporting evidence for solar flare effects in the $F$ region of the ionosphere, R. W. Knecht and K. Davies, Nature 192, No. 4800, 348-350 (Oct. 28, 1961).

Measurement of effect of moisture on heat transfer through insulated flat-roof constructions, F. J. Powell and H. E. Robinson, Am. Soc. Testing Materials Spec. Tech. Publ. 312, 35-66 (1961)

Dependence of interval between flare and associated sudden commencement storm on prestorm conditions, M. W. Haurwitz, J. Geophys. Res. Letter 67, 2979-2982 (July 1962).

The application of NMR in determination of the structure of cyclanols. I. The structures of cyclohexane-1,3-diols, H. Finegold and H. Kwart, J. Organic Chem. 27, 2361-2365 (1962)

APPA-TAPPI reference material program. I. Interlaboratory investigation of TAPI standard T $414 \mathrm{~m}-49$, internal tearing resistance of paper, T. W. Lashof, Tappi 45, No. 8, 656 (1962)

Spectrophotofluorometric studies of degraded cotton cellulose, S. D. Toner and K. F. Plitt, Tappi 45, No. 8, 681-688 (Aug. 1962).

Properties of dental amalgams made from spherical alloy particles, N. C. Demaree and D. F. Taylor, J. Dental Res. 41, No. 4, 890-906 (July-Aug. 1962).

Emission spectra of solids condensed at very low temperatures from the electrical discharge products of nitrogen and carbon monoxide or acetylene, S. L. N. G. Krishnamachari, R. W. Nicholls, and H. P. Broida, Proc. Indian Acad. Sci. LIV, 61-68 (1961).

Excitation of the red and green coronal lines, C. Pecker and R. N. Thomas, Ann. Astrophys. 25, 100-108 (Mar.-Apr. 1962).

Dependence of the ionospheric $F$ region on the solar cycle, J. W. Wright, Nature 194, No. 4827, 461-462 (May 1962).

Fast-melting alloy forms water jacket for small klystrons, W. J. Anson and E. Niesen, Electron. Design, pp. 42-45 (Mar. 1962)

Boolean matrix equations in digital circuit design, R. S. Ledley, IRE Trans. Electronic Computers EC-8, No. 2, 131-139 (June 1959).

Spectrophotometric determination of hydroperoxide in diethyle ether, W. C. Wolfe, Anal. Chem. 34, No. 10, 1328-1330 (Sept. 1962).

Exchange behavior of kaolins of varying degrees of crystallinity, W. C. Ormsby, J. M. Shartsis and K. H. Woodside, J. Am. Ceram. Soc 45, No. 8, 361-366 (Aug. 1962). 
Analysis of the hydroxyl radical vibration rotation spectrum between $3900 \mathrm{~A}$ and $15000 \mathrm{~A}$, A. M. Bass and D. Garvin, J. Mol. Spectry. 9, No. 2, 114-123 (Aug. 1962).

Microwave measurements in the NBS electronic calibration center, R. E. Larson, Inst. Elec. Engrs. 109, pt. B, Suppl. No. 23, 644-650 (1962).

Structure and structure imperfections of solid $\beta$-oxygen, E. M. Horl, Acta. Cryst. 15, No. 9, 845-850 (Sept. 1962).

Kinetics of $\mathrm{Cs}^{+}$desorption from tungsten, M. D. Scheer and J. Fine, J. Chem. Phys. 37, No. 1, 107-113 (July 1962).

Effect of additives on silver iodide particles exposed to light, G. Burley and D. W. Herrin, J. Appl. Meteorol. 1, No. 3, 355-356 (Oct. 1962).

A modulated sub-carrier technique of measuring microwave attenuation, G. E. Schafer and R. R. Bowman, Inst. Elec, Engrs. 109, pt. B, Suppl. 23, 783-786 (1962).

A survey of microwave power-measurement techniques employed at the National Bureau of Standards, G. F. Engen, Inst. Elec. Engrs. 109, pt. B, Suppl. No. 23, 734-739 (1962).

Symposium on teaching of materials: Aspects of material behavior significant to engineers, J. M. Frankland, J. Eng.
Mech. Div.-Proc. Am. Soc. Civil Engr. Paper No. 3246, 75-81 (Aug. 1962).

Neutral meson photoproduction from complex nuclei, R. A. Schrack, J. E. Leiss and S. Penner, Phys. Rev. 127, No. 5, 1772-1783 (Sept. 1, 1962).

Excitation of modes at very low frequency in the earthionosphere wave guide, J. R. Wait, J. Geophys. Res. 67, No. 10, 3823-3828 (Sept. 1962).

The growth and roots of electronic computers, J. L. Little, Student Quart. and EE Digest, Joint Publ. of Am. Inst. Elec. Engr. and IRE 1, No. 1, 64-70 (Sept. 1962).

Acoustical interferometer employed as an instrument for measuring low absolute temperatures, G. Cataland and H. H. Plumb, J. Acoust, Soc. Am. 34, No. 8, 1145-1146 (Aug. 1962).

* Publications for which a price is indicated are available from the Superintendent of Documents, U.S. Government Printing Office, Washington 25, D.C. (foreign postage one-fourth additional). Reprints from outside journals and the NBS Journal of Research may often be obtained directly from the authors. 